\title{
GIBBS STATE UNIQUENESS FOR ANHARMONIC QUANTUM CRYSTAL WITH A NONPOLYNOMIAL DOUBLE-WELL POTENTIAL
}

\author{
Alexei L. REBENKO ${ }^{1}$ and Valentin A. ZAGREBNOV ${ }^{2}$ \\ Centre de Physique Théorique ${ }^{3}$, CNRS-Luminy, Case 907 \\ F-13288 Marseille - Cedex 9, France
}

\begin{abstract}
We construct the Gibbs state for $\nu$-dimensional quantum crystal with site displacements from $\mathbb{R}^{d}, d \geq 1$, and with a one-site non-polynomial double-well potential, which has harmonic asymptotic growth at infinity. We prove the uniqueness of the corresponding Euclidean Gibbs measure (EGM) in the lightmass regime for the crystal particles. The corresponding state is constructed via a cluster expansion technique for an arbitrary temperature $T \geq 0$. We show that for all $T \geq 0$ the Gibbs state (correlation functions) is analytic with respect to external field conjugated to displacements provided that the mass of particles $m$ is less than a certain value $m_{*}>0$. The high temperature regime is also discussed.
\end{abstract}

Keywords : quantum crystal model, Gibbs state, Euclidean Gibbs measures, quantum fluctuations, light-mass regime, cluster expansions.

Mathematics Subject Classification : 60H30, 82B31

\footnotetext{
${ }^{1}$ On leave of absence from Institute of Mathematics, Kiev, Ukraine

${ }^{2}$ Université de la Méditerranée (Aix-Marseille II)

${ }^{3}$ Unité Propre de Recherche 7061
} 


\section{Introduction}

It is generally excepted that for investigation of different physical phenomena in quantum crystals one can consider an infinite system of interacting anharmonic oscillators, which are situated in the sites of $\nu$-dimensional lattice $\mathbb{Z}^{\nu}$. The heuristic Hamiltonian of such a system (in the case of 2-body interaction) has the following form:

$$
H=-\frac{\hbar^{2}}{2 m} \sum_{j \in \mathbb{Z}^{\nu}} \Delta_{j}+\sum_{j \in \mathbb{Z}^{\nu}} W\left(q_{j}\right)+\frac{J}{2} \sum_{i, j \in \mathbb{Z}^{\nu}, i \neq j} Q\left(q_{i}, q_{j}\right)-\sum_{j \in \mathbb{Z}^{\nu}} h q_{j},
$$

where $m$ is the mass of particles, the operator $\Delta_{j}$ corresponds the kinetic energy of the system and, in fact, is $d$-dimensional Laplace operator in the one-particle Hilbert space $L^{2}\left(\mathbb{R}^{d}, d q\right)$, where $d q$ is the Lebesgue measure on $\mathbb{R}^{d}, q_{j} \in \mathbb{R}^{d}$ for $j \in \mathbb{Z}^{\nu}$ is displacement of a particle from its position in the site $j \in \mathbb{Z}^{\nu}$. For general case $d \leq \nu \geq 1$. But the most of results, which where obtained earlier are for $d=1$. The particles are confined near their sites by potential $W\left(q_{j}\right)$. A harmonic one-site potential $W_{\text {harm }}\left(q_{j}\right)=$ $\frac{1}{2} a q_{j}^{2}, a>0$ together with a harmonic two-particle interaction $Q(\cdot, \cdot)$ define a well-known harmonic crystal model (1.1). To produce a model describing a (ferroelectric) structural phase transition one usually takes for $W(\cdot)$ a doublewell anharmonic potential, keeping $Q(\cdot, \cdot)$ harmonic (see e.g. [1,19,24]). For example,

$$
W(q)=\frac{1}{4} b\left(q^{2}\right)^{2}-\frac{1}{2} a q^{2}, \quad a, b \in \mathbb{R}_{+}^{1},
$$

or semibounded from below polynomials of higher degree with more than two equal minima. Here $q^{2} \equiv q \cdot q$ is the scalar square of displacement vector $q \in \mathbb{R}^{d}$. The proof of existence of phase transition in such kind of systems for harmonic interaction $Q\left(q_{i}, q_{j}\right)$ were obtained in $[12,15,26,34,40,47,53]$ for the case

$$
Q\left(q_{i}, q_{j}\right)=Q_{i j}\left(q_{i}-q_{j}\right)^{2}
$$

where $\hat{Q}=\left(Q_{i j}\right)_{i, j \in \mathbb{Z}^{\nu}}$ is a matrix of non-negative harmonic-force constants. The term $h \cdot q_{j}=\sum_{\alpha=1}^{d} h^{\alpha} q_{j}^{\alpha}$ break the symmetry in the direction of the 
external field $h$. The non-uniqueness of Gibbs states for $h=0$ is proved when the mass of the particles $m$ is sufficiently large and the temperature $T$ is sufficiently low, or $\beta=(k T)^{-1}$ is sufficiently large.

From the other side, another interesting phenomenon, the suppression of the long-range order by strong quantum fluctuations in such systems was experimentally observed (see, e.g. [55]) and was discussing long time ago from the physical point of view, see [51], or the books [1,19]. A rigorous study of this phenomenon was given in the series of papers. For the $d=1$ displacement and all $\nu \geq 1$ the suppression of the order parameter for all temperatures including $T=0$ (as soon as the particle mass is smaller than some threshold: $m<m_{*}$ ) was proved in [58]. Later it was shown in [3] for $d=1$ and in [37-39] for $d \in \mathbb{N}$, that not only the long-range order but also any critical behaviour of displacements of particles from the equilibrium positions are suppressed at all temperatures $T>0$ if the model is "strongly quantum", i.e., if the mass $m$ is small enough. But a more important problem is to prove the uniqueness of the Gibbs state for such systems for sufficiently small mass of particles. It would imply the absence of all critical anomalies in the regime of "strong quantumness". The first step in this direction was made in [44] for $d=1$, where for small values of $m$ the convergence of corresponding cluster expansions was proved for all values of temperature $T \geq 0$. See also [8], where the cluster expansion is constructed by another and simpler technique but for small $m$, which depends on the positive $T>0$. The result of [44] has been extended to the multi-dimensional spin in [27]. This in particular implies that the order parameter is always zero if $m<m_{*}$ for any values of $T \geq 0$ [43]. It gives, also, uniqueness of the EGM (see $[2,31,33]$ and [7] for detail description of the Euclidean approach) at least for so-called "compact" boundary conditions for displacements. In particular, this is true if displacements are reduced to a compact (so-called compact spins). This resumes the results of [44] and [43] about the suppression of the displacement phase transition by the large quantum fluctuations in small masse domain $m<m_{*}$. For models with the compact spin the uniqueness was proved in [9]. Then in [4] the uniqueness of EGM was proved for $d=1$ and for every fixed inverse temperature $\beta<\infty$ if the mass is less than some threshold: $m<m_{*}(\beta)$. Finally, the dependence of the mass threshold on the temperature was eliminated in recent papers [5],[6] for the set of tempered EGM. 
Remark 1.1 These results were obtained for the case of polynomial anharmonic one-site potential of the form:

$$
W(q)=v\left(q^{2}\right)=\sum_{p=1}^{s} a_{2 p}\left(q^{2}\right)^{p}, \quad a_{2 s}>0, \quad s>1, \quad s \in \mathbb{N} .
$$

with additional assumption on $W(q)$ that $v(\cdot)$ is convex on $\mathbb{R}_{+}^{1}$. For the proof of these results the fact of smallness of the parameter $m^{(s-1) /(s+1)}$ for small $m$, i.e. $s>1$, is crucial. Recall that in this case, after an appropriate unitary transformation of the Hamiltonian, the parameter $m^{(s-1) /(s+1)}$ stands in front of the harmonic interaction term $Q$. So the light-mass regime corresponds to the one-site energy domination and the weak interaction of anharmonic oscillators defined by $W$, that implies the uniqueness of the EGM.

On the other hand, let us define $s \geq 0$ by

$$
s=\inf _{\alpha \geq 0}\left\{\alpha: \limsup _{q^{2} \rightarrow \infty} v\left(q^{2}\right) /\left(q^{2}\right)^{\alpha}<\infty\right\} .
$$

If $s \leq 1$, then in the light-mass domain ("strong quantumness") the parameter $m^{(s-1) /(s+1)}$ is not small, i.e. the approach developed for $s>1$ does not work. So, the rate of the growth of anharmonic potential is an important parameter distinguishing different regimes, see discussion in [43] and [58].

Remark 1.2 If $s<1$, then in the light-mass limit the harmonic interaction term dominates the one-site anharmonic potential, i.e. the system has to behave close to a Debye harmonic crystal (not proved). In the marginal case $s=1$ the both harmonic interaction and harmonic part of $W$ dominate the anharmonicity. From the physical point of view the large-distant behaviour of the one-site anharmonic potential is not well-justified. So, a priori all above scenario are possible.

For example, the paper [54] proposed a model with the one-site non-polynomial anharmonic potential of the form:

$$
W(q)=\frac{1}{2} a q^{2}+b e^{-\delta q^{2} / 2}, \quad a, b, \delta \in \mathbb{R}_{+}^{1} .
$$

corresponding to $s=1$. It is easy to see that for $a<b \delta$ the minimum of the potential (1.5) is not unique and the system may undergo a displacement 
phase transition. For the case of so-called self-consistent phonon approximation [1], or for the displacement quantum spherical model it was proved in [48] for $(1.3)$ and in [54,58] for (1.5). In general case this follows from [15], see also [47]. On the other hand, in [48] and [54,58] it is shown that there is a non-zero threshold mass $m_{*}$ such that the phase transition in the displacement quantum spherical model is suppressed by the "strong quantumness" as soon as $m<m_{*}$.

The aim of the present paper is study the case of the quadratic growth, $s=1$, of the double-well anharmonic one-site potential $W(1.5)$. We prove that (similar to the case $s>1$ ) there is a non-zero mass $m_{*}$ such that in the light-mass domain: $m<m_{*}$ the critical behaviour of the quantum anharmonic system (1.1) for $h=0$ is suppressed for arbitrary dimension of particles displacements $d \geq 1$ and $\nu \geq 3$. More precisely, we prove the uniqueness of the corresponding Gibbs state. Our result is based on the proof of convergence of the cluster expansions for the quantum Euclidean Gibbs Measure in the thermodynamic limit for arbitrary temperature $T \geq 0$ and sufficiently small mass $m<m_{*}$ of particles (uniformly in temperature). Notice that since for $s=1$ the interaction in the light-mass limit $m<m_{*}$ is not negligible (Remark 1.2), our strategy is different to that for $s>1$, cf [44]. In that sense our result supplements the recent proof of the Gibbs state uniqueness for $s>1$, see $[5,6]$.

Now there are few other remarks in order to outline our strategy of the proof the uniqueness for the case $s=1$.

The anharmonic perturbation in (1.5) can be re-written in the following form

$$
V(q)=b e^{-\delta q^{2} / 2}=b \int d \mu(\alpha) e^{i \sqrt{\delta} \alpha \cdot q},
$$

where $d \mu(\alpha)=(2 \pi)^{-d / 2} e^{-\alpha^{2} / 2} d \alpha$ is the Gaussian measure on $\mathbb{R}^{d}$. So anharmonic term (1.6) is a gentle perturbation of the quantum harmonic system in the sense of Albeverio-Høegh-Krohn [2]. Unfortunately, our situation is similar to [2] only at the first glance. In fact, there are some essential differences. In [2] the bounded measure $d \mu$ has to have a compact support. Under this condition the authors managed to apply the Kirkwood-Salsburg Equation (KSE) technique to prove the existence of the corresponding correlation functions in thermodynamic limit as well as their analyticity in coupling constant $\lambda$ (in our case $\lambda \equiv b$ ) in some domain $|\lambda|<\lambda_{0}$ for all temperatures. 
We can not apply this technique, since of the Gaussian measure in (1.6) has infinite support in $\mathbb{R}^{d}$. By consequence it is impossible to find a Banach space $E_{\xi}$ for correlation functions and the corresponding KSE. As it was indicated in [2], Remark 2, for extension of the results to the measure with unbounded support one needs a modification of the Banach space. But even that is not essential, since the main difficulty for $s=1$ is connected with the parameter of analyticity. Instead of $\lambda$ we have as the small parameter the particle mass $m$, which is involved in the Hamiltonian via the kinetic-energy term, see (1.1). On the other hand it is impossible to expect the analyticity in domain $|m|<m_{*}$, because we must always consider Rem $>0$ to define the system (1.1).

We overcome these difficulties by a cluster expansions technique. The choice of the cluster expansions is defined by the dependence of the parameters in the Hamiltonian on the mass $m$ after an appropriate rescaling, see (2.6). In the case of anharmonic potential with harmonic asymptotics $s=1$ the dependence on the mass $m$ remains only in the rescaled temperature and in the rescaled anharmonic potential (1.5). Therefore, the expansions should be arranged with respect to (w.r.t.) the anharmonic part of the one-site potential. (Recall that for $s>1$ the corresponding cluster expansions are constructed w.r.t. two-body harmonic interaction.) This gives a possibility to construct the limiting Gibbs state and to prove its analyticity in some small circle $\left|m-m_{*} / 2\right|<m_{*} / 2$ of the complex values of $m$ around the point $m_{*} / 2>0$.

To prove the uniqueness of the corresponding EGM we profit the Theorem 3.1 of [6] and we use the cluster property of the EGM moments, which is a consequence of the cluster expansion convergence established in the present paper. Similar to [43] we prove the analyticity of the state w.r.t. the external field $h$. As a consequence this immediately implies that the displacement order parameter (in the region of convergence of the cluster expansions) is equal to zero for all temperatures $T \geq 0$ as soon as $m<m_{*}$.

A brief contents of this paper is the following. In Section 2 we describe our system (1.5) in more details and we formulate the main results. In Section 3 we construct the cluster expansions w.r.t. the anharmonic part of the one-site potential, and we give the proof of main theorems. In Section 4 we provide all necessary estimates. Section 5 is devoted to the limiting Gibbs state analyticity w.r.t. to the external field, and in Section 6 we briefly discuss the proof of the convergence of cluster expansions and the uniqueness in the 
case of high temperatures.

\section{Description of the system and main results}

We consider a $\nu(\geq 3)$-dimensional quantum crystal model with $d(\geq 1)$ component site displacements (or d-dimensional continuous infinite-spin variables) on the cubic lattice $\mathbb{Z}^{\nu}$. With each site $j \in \mathbb{Z}^{\nu}$ we associate a oneparticle physical Hilbert space $L^{2}\left(\mathbb{R}^{d}, d q\right)$, where $d q$ is the Lebesgue measure on $\mathbb{R}^{d}$. Then

$$
\begin{gathered}
\mathcal{H}_{\Lambda}=\bigotimes_{j \in \Lambda} L^{2}\left(\mathbb{R}^{d}, d q_{j}\right) \simeq L^{2}\left(\mathbb{R}^{d|\Lambda|}, d q_{\Lambda}\right), \\
d q_{\Lambda}=\prod_{j \in \Lambda} d q_{j},
\end{gathered}
$$

is the Hilbert space related to some given bounded set $\Lambda \subset \mathbb{Z}^{\nu}$, with number of sites $|\Lambda|<\infty$.

For a finite set $\Lambda \subset \mathbb{Z}^{\nu}$ we consider in $\mathcal{H}_{\Lambda}$ the Hamiltonian:

$$
H_{\Lambda}=H_{0}^{\Lambda}+V^{\Lambda} \text {. }
$$

Here the harmonic part of the Hamiltonian has the form:

$$
H_{0}^{\Lambda}=-\frac{1}{2 m} \sum_{j \in \Lambda} \Delta_{j}+\frac{1}{2} a \sum_{j \in \Lambda} q_{j}^{2}+\frac{J}{4} \sum_{\langle i, j\rangle \subset \Lambda}\left(q_{i}-q_{j}\right)^{2}+C_{\Lambda},
$$

where $m$ is the mass of particles (we put $\hbar=1$ ), $\Delta_{j}$ is the Laplace operator in $L^{2}\left(\mathbb{R}^{d}, d q_{j}\right)$, the sum is extended over all pairs $\langle i, j\rangle \subset \Lambda$ for which $\|i-j\|=1$ and $J>0$. We consider a nearest-neighbors interaction only for simplicity. The constant $C_{\Lambda}$ we will be chosen later.

We put a one-site potential $W(q)$ in (1.1) to be equal to $(1.5)$ for $q^{2}=q \cdot q$, i.e., for $q \in \mathbb{R}^{d}$. So the anharmonic part of the one-site potential in (2.1) has the form

$$
V(q)=b e^{-\delta q^{2} / 2}, \quad b \geq 0, \delta \geq 0, \quad q \in \mathbb{R}^{d} .
$$

To prove the existence of the Gibbs states in the light-mass regime for small temperatures, including $T=0$, we need a supplementary argument 
based on the cluster expansions. Below we do this first for the periodic boundary conditions (p.b.c.), then we complete our construction by the case of the empty boundary conditions The last we use later to pass to tempered non-empty boundary conditions Notice that the cluster expansions for p.b.c. prove also the clustering property of the corresponding EGM for $m<m_{*}$ and for any $T \geq 0$. Therefore, simultaneously with existence we get uniqueness of translation invariant EGM for all temperature, including $T=0$, as soon as the particle mass is light enough: $m<m_{*}$. So, first we assume in (2.1), (2.2) the p.b.c.:

$$
\Lambda=\left\{j \in \mathbb{Z}^{\nu} \mid-N_{\mu} / 2<j^{(\mu)} \leq N_{\mu} / 2, \mu=1, \ldots, \nu ; N_{\mu} / 2 \in \mathbb{N}\right\} .
$$

Thus, $|\Lambda|=N_{1} \cdots N_{\nu}$ and

$$
q_{\left(j^{(1)}, \ldots, j^{(\mu)}+N_{\mu}, \ldots, j^{(\nu)}\right)}^{(\alpha)}=q_{j}^{(\alpha)}, \quad \alpha=1, \ldots, d, \quad \mu=1, \ldots, \nu .
$$

Then, to complete the proof of the uniqueness of the EGM, in the next section we consider also a general boundary conditions generated by some fixed configuration $\bar{\omega}$ from the set $\tilde{\Omega}_{\hat{\beta}}^{t}$, which includes so-called tempered configurations, see $[6,13,14]$ and $[46]$.

Let $\mathcal{L}\left(\mathcal{H}_{\Lambda}\right)$ be algebra of bounded operators on $\mathcal{H}_{\Lambda}$. We consider the temperature quantum Gibbs state on $\mathcal{L}\left(\mathcal{H}_{\Lambda}\right)$ defined by

$$
\rho_{\Lambda}^{\beta}(A)=\frac{\operatorname{Tr}\left(A e^{-\beta H_{\Lambda}}\right)}{Z_{\beta}(\Lambda)}, \quad \beta=\frac{1}{k_{B} T},
$$

where $Z_{\beta}(\Lambda)=\operatorname{Tr} e^{-\beta H_{\Lambda}}$ and $A \in \mathcal{L}\left(\mathcal{H}_{\Lambda}\right)$.

Note, that the algebras $\mathcal{L}\left(\mathcal{H}_{\Lambda}\right)$ (for different $\Lambda$ ) are isometrically embedded into $\mathcal{L}\left(\mathcal{H}_{\Lambda^{\prime}}\right)$ for $\Lambda \subset \Lambda^{\prime}$. Using this fact we can define the inductive limit

$$
\mathfrak{A}_{0}=\lim _{\Lambda \nearrow \mathbb{Z}^{\nu}} \mathcal{L}\left(\mathcal{H}_{\Lambda}\right)
$$

which a normed $*$ - algebra of local observables. The closure of this algebra in the operator norm forms the algebra of bounded quasi-local observables

$$
\mathfrak{A}=\overline{\mathfrak{A}}_{0} .
$$

The main result of the present paper is the following theorem. 
Theorem 2.1 For the system of quantum particles with Hamiltonian (2.1)(2.3) there is some $m_{*}>0$, such that for any $0<m<m_{*}$ the limiting Gibbs state

$$
\lim _{\Lambda \nearrow \mathbb{Z}^{\nu}} \rho_{\Lambda}^{\beta}(A)=\rho^{\beta}(A), \quad A \in \mathfrak{A}_{0},
$$

- exists for all $T \geq 0$;

- it is a unique translation invariant quantum Gibbs state (corresponding to $E G M)$ on the algebra $\mathfrak{A}_{0}$, which can be by continuity extended to the algebra $\mathfrak{A}$.

Notice that by virtue of results of the paper [32](see also $[7,33,44])$ it is sufficient to prove this theorem for some sub-algebra $\mathfrak{A}^{\tau} \subset \mathfrak{A}$ of local operators, which we describe below. The main technical tools are the Feynman-Kac formula and the representation of the Gibbs states (2.5) on this algebra by functional integrals with respect to some measure $\mu(\cdot)$ which we are going to construct.

Since we are going to consider domain of small masses, the following standard light-mass rescaling of variables (appeared for the first time in this context in [58]) is relevant:

$$
q_{j}=\alpha x_{j}, \quad \alpha=m^{-\frac{1}{4}} .
$$

This change of variables induces the unitary map :

$$
\begin{gathered}
U: \mathcal{H}_{\Lambda} \rightarrow \mathcal{H}_{\Lambda} \\
(U f)\left(x_{\Lambda}\right)=\alpha^{|\Lambda| / 2} f\left((\alpha x)_{\Lambda}\right), \quad(\alpha x)_{\Lambda}=\left\{\alpha x_{j}, j \in \Lambda\right\} .
\end{gathered}
$$

One can check that

$$
U H_{\Lambda} U^{-1}=m^{-\frac{1}{2}} \hat{H}_{\Lambda},
$$

where

$$
\begin{gathered}
\hat{H}_{\Lambda}=\hat{H}_{0}^{\Lambda}+\hat{V}^{\Lambda} \\
\hat{H}_{0}^{\Lambda}=-\frac{1}{2} \sum_{j \in \Lambda} \Delta_{x_{j}}+\frac{1}{2} a \sum_{j \in \Lambda} x_{j}^{2}+\frac{J}{4} \sum_{\langle i, j\rangle \subset \Lambda}\left(x_{i}-x_{j}\right)^{2}+C_{m}^{\Lambda}, \\
C_{m}^{\Lambda}=C_{\Lambda} m^{1 / 2},
\end{gathered}
$$




$$
\hat{V}^{\Lambda}=b_{m} \sum_{j \in \Lambda} e^{-\delta_{m} x_{j}^{2} / 2}, \quad b_{m}=b m^{1 / 2}, \quad \delta_{m}=\delta m^{-1 / 2} .
$$

We also define a rescaled inverse temperature

$$
\hat{\beta}=\beta m^{-\frac{1}{2}},
$$

which yields

$$
U \beta H_{\Lambda} U^{-1}=\hat{\beta} \hat{H}_{\Lambda} .
$$

Then the Gibbs state (2.5) takes the form:

$$
\rho_{\Lambda}^{\beta}(A)=\hat{\rho}_{\Lambda}^{\hat{\beta}}(\hat{A}), \quad \hat{A}=U A U^{-1},
$$

where

$$
\hat{\rho}_{\Lambda}^{\hat{\beta}}(\cdot)=\hat{Z}_{\hat{\beta}}^{-1}(\Lambda) \operatorname{Tr}\left\{\cdot \exp \left(-\hat{\beta} \hat{H}_{\Lambda}\right)\right\} .
$$

To represent the state $(2.12)$ in the form of the functional integral we rewrite the operator $\hat{H}_{0}^{\Lambda}$ as follows

$$
\begin{gathered}
\hat{H}_{0}^{\Lambda}=\sum_{\alpha=1}^{d} \hat{H}_{0}^{(\alpha), \Lambda}, \\
\hat{H}_{0}^{(\alpha), \Lambda}=-\frac{1}{2} \Delta_{\Lambda}^{(\alpha)}+\frac{1}{2}\left(x_{\Lambda}^{(\alpha)}, B_{\Lambda}^{2} x_{\Lambda}^{(\alpha)}\right)-\frac{1}{2} \operatorname{Tr} B_{\Lambda},
\end{gathered}
$$

where

$$
\Delta_{\Lambda}^{(\alpha)}=\sum_{j \in \Lambda} \frac{\partial^{2}}{\partial x_{j}^{(\alpha)^{2}}}, \quad B_{\Lambda}^{2}=-J \hat{\Delta}_{\Lambda}+a \mathbb{1}_{\Lambda},
$$

$x_{\Lambda}^{(\alpha)}=\left(x_{j}^{(\alpha)}\right)_{j \in \Lambda} \in \mathbb{R}^{|\Lambda|}$, and $B_{\Lambda}^{2}$ is a periodic $|\Lambda| \times|\Lambda|$ matrix, defined by the elements:

$$
\left(-\hat{\Delta}_{\Lambda}\right)_{j k}= \begin{cases}2 \nu, & \text { for } j=k \\ -1, & \text { for }|j-k|=1 \\ 0, & \text { otherwise }\end{cases}
$$


and

$$
(\mathbb{1})_{j k}=\delta_{j k}=\delta_{j^{(1)} k^{(1)}} \cdots \delta_{j^{(\nu)} k^{(\nu)}} .
$$

Now we fix the choice of the constant $C_{\Lambda}$ in $(2.2),(2.9)$ by

$$
C_{\Lambda}=\frac{d}{2 \sqrt{m}} \operatorname{Tr} B_{\Lambda}
$$

By virtue of p.b.c. the lattice operator $B_{\Lambda}$ is cyclic (see (2.16) and [30, Sec.16.4]), so we can calculate its eigenvalues explicitly:

$$
\lambda_{k}=\sqrt{\varepsilon(k)}, \quad \varepsilon(k)=a+4 J \sum_{\mu=1}^{\nu} \sin ^{2}\left(k^{(\mu)} / 2\right), \quad k \in \Lambda^{*},
$$

where the set $\Lambda^{*}$ is dual to $\Lambda$, i.e.

$$
\Lambda^{*}=\left\{k \in \mathbb{R}^{\nu} \mid k=\left\{k^{(\mu)}\right\}_{\mu=1}^{\nu}, k^{(\mu)}=\frac{2 \pi}{N_{\mu}} n^{(\mu)}, n^{(\mu)}=0, \pm 1, \ldots, \pm N_{\mu}-1 / 2, N_{\mu} / 2\right\} .
$$

Now the Feynman-Kac formula (see e.g. [50],[52]) gives

$$
\begin{gathered}
\operatorname{Tr} e^{-\hat{\beta} \hat{H}_{\Lambda}}=\int_{\mathbb{R}^{d|\Lambda|}} d x_{\Lambda}\left(e^{-\hat{\beta} \hat{H}_{\Lambda}}\right)\left(x_{\Lambda} ; x_{\Lambda}\right)= \\
=\int_{\mathbb{R}^{d|\Lambda|}} d x_{\Lambda} \int_{\Omega_{\hat{\beta}, \Lambda}} W_{x_{\Lambda} ; x_{\Lambda}}^{\hat{\beta}}\left(d \omega_{\Lambda}\right) e^{-\int_{0}^{\hat{\beta}} d \tau \hat{U}\left(\omega_{\Lambda}(\tau)\right)},
\end{gathered}
$$

with

$$
\hat{U}\left(x_{\Lambda}\right)=\frac{1}{2}\left(x_{\Lambda}, B_{\Lambda}^{2} x_{\Lambda}\right)-\frac{d}{2} \operatorname{Tr} B_{\Lambda}+\hat{V}\left(x_{\Lambda}\right),
$$

where we put $\hat{V}\left(x_{\Lambda}\right)=\hat{V}^{\Lambda}$, see $(2.10)$, and

$$
W_{x_{\Lambda} ; x_{\Lambda}}^{\hat{\beta}}\left(d \omega_{\Lambda}\right)=\prod_{j \in \Lambda} W_{x_{j} ; x_{j}}^{\hat{\beta}}\left(d \omega_{j}\right),
$$


here $W_{x_{j} ; x_{j}}^{\hat{\beta}}\left(d \omega_{j}\right)$ is the conditional Wiener measures on the space of continuous periodic trajectories

$$
\begin{gathered}
\Omega_{\hat{\beta}, \Lambda}=\left\{\omega_{\Lambda}(\cdot) \mid \omega_{\Lambda}: S_{\hat{\beta}} \rightarrow \mathbb{R}^{d|\Lambda|}\right\}, \quad S_{\hat{\beta}}:=[0, \hat{\beta}], \\
\omega_{\Lambda}(\cdot)=\left\{\omega_{j}(\cdot), j \in \Lambda \mid \omega_{j} \in \Omega_{\hat{\beta}}, \Omega_{\hat{\beta}}:=C\left(S_{\hat{\beta}} \rightarrow \mathbb{R}^{d}\right)\right\} .
\end{gathered}
$$

Now, following [2] the unperturbed measure in the right-hand side of (2.20) (i.e. $\hat{V}^{\Lambda}=0$ ) can be rewritten with the help of a Gaussian integral over the Hilbert space:

$$
\mathcal{H}_{\hat{\beta}, \Lambda}:=\left\{\omega_{\Lambda} \in\left(\Omega_{\hat{\beta}, \Lambda}, \Sigma_{\hat{\beta}, \Lambda}\right) \mid\left\|\omega_{\Lambda}\right\|^{2}<\infty\right\} .
$$

Here $\Sigma_{\hat{\beta}, \Lambda}$ is the standard $\sigma$-algebra of $\Omega_{\hat{\beta}, \Lambda}$-subsets generated by Borel cylinder subsets and norm is defined by

$$
\left\|\omega_{\Lambda}\right\|^{2}=\int_{0}^{\hat{\beta}} d \tau\left[\left(\frac{d \omega_{\Lambda}(\tau)}{d \tau}, \frac{d \omega_{\Lambda}(\tau)}{d \tau}\right)+\left(\omega_{\Lambda}(\tau), \omega_{\Lambda}(\tau)\right)\right],
$$

where $(\cdot, \cdot)$ is the scalar product in $l_{2}\left(\mathbb{R}^{d|\Lambda|}\right)$. Then for any real continuous function $F$ defined on $\mathcal{H}_{\hat{\beta}, \Lambda}$ with this unperturbed measure one gets:

$$
\begin{gathered}
\int_{\mathbb{R}^{d|\Lambda|}} d x_{\Lambda} \int_{\Omega_{\hat{\beta}, \Lambda}} W_{x_{\Lambda} ; x_{\Lambda}}^{\hat{\beta}}\left(d \omega_{\Lambda}\right) e^{-\frac{1}{2} \int_{0}^{\hat{\beta}} d \tau\left(\omega_{\Lambda}(\tau), B_{\Lambda}^{2} \omega_{\Lambda}(\tau)\right)+\frac{d}{2} \operatorname{Tr} B_{\Lambda}} F\left(\omega_{\Lambda}\right)= \\
=C_{0} \int_{\mathcal{H}_{\hat{\beta}, \Lambda}} d \mu_{0}^{\Lambda}\left(\phi_{\Lambda} \mid p\right) F\left(\phi_{\Lambda}\right)=C_{0} \int_{\mathcal{H}_{\hat{\beta}, \Lambda}} d \mu_{0}^{\Lambda}\left(\phi_{\Lambda}\right) F\left(\phi_{\Lambda}\right),
\end{gathered}
$$

where $C_{0}$ is a normalization constant and $d \mu_{0}^{\Lambda}\left(\phi_{\Lambda} \mid p\right)$ is the Gaussian measure on $\mathcal{H}_{\hat{\beta}, \Lambda}$ which corresponds to p.b.c.. We skip the index $p$ in the following. By (2.20) and (2.23) it is clear that

$$
C_{0}=Z_{\hat{\beta}}^{0}(\Lambda)=\operatorname{Tr} e^{-\hat{\beta} \hat{H}_{0}^{\Lambda}} .
$$

Hence the right-hand side of (2.23) is the expectation:

$$
\langle F\rangle_{\mu_{0}^{\Lambda}}=\int_{\mathcal{H}_{\hat{\beta}, \Lambda}} d \mu_{0}^{\Lambda}\left(\phi_{\Lambda}\right) F\left(\phi_{\Lambda}\right)
$$


with respect to the Gaussian measure on the Hilbert space of continuous periodic functions generated by the corresponding Gaussian process with the zero mean and the covariance $G_{0}^{\Lambda}$ :

$$
G_{0 ; j k}^{\Lambda ; \alpha \alpha^{\prime}}\left(\tau-\tau^{\prime}\right)=\int_{\mathcal{H}_{\hat{\beta}, \Lambda}} d \mu_{0}^{\Lambda}\left(\phi_{\Lambda}\right) \phi_{j}^{(\alpha)}(\tau) \phi_{k}^{\left(\alpha^{\prime}\right)}\left(\tau^{\prime}\right)=\delta_{\alpha \alpha^{\prime}} G_{0 ; j k}^{\Lambda}\left(\tau-\tau^{\prime}\right)
$$

Here $\alpha, \alpha^{\prime} \in\{1,2, \ldots, d\}$, and $G_{0 ; j k}^{\Lambda}\left(\tau-\tau^{\prime}\right)$ is the Green function of the operator $\left(-\frac{d^{2}}{d \tau^{2}}\right) \mathbb{1}_{\Lambda}+B_{\Lambda}^{2}$ on $L_{2}\left(\Lambda \otimes S_{\hat{\beta}} ; \mathbb{R}^{d}\right)$ with p.b.c. More explicitly [50]:

$$
G_{0 ; j k}^{\Lambda}\left(\tau-\tau^{\prime}\right)=\frac{1}{|\Lambda|} \sum_{l \in \Lambda^{*}} e^{i(j-k) \cdot l} \frac{1}{\hat{\beta}} \sum_{n \in \mathbb{Z}^{1}} \frac{\cos \left(2 \pi\left(\tau-\tau^{\prime}\right) n / \hat{\beta}\right)}{\left(4 \pi^{2} n^{2} / \hat{\beta}^{2}\right)+\varepsilon(l)}
$$

or taking the sum over $n$, we get (see e.g.[50])

$$
G_{0 ; j k}^{\Lambda}\left(\tau-\tau^{\prime}\right)=\frac{1}{|\Lambda|} \sum_{l \in \Lambda^{*}} e^{i(j-k) \cdot l} \frac{e^{\left(\hat{\beta}-\left|\tau-\tau^{\prime}\right|\right) \sqrt{\varepsilon(l)}}+e^{\left|\tau-\tau^{\prime}\right| \sqrt{\varepsilon(l)}}}{2 \sqrt{\varepsilon(l)}\left(e^{\hat{\beta} \sqrt{\varepsilon(l)}}-1\right)} .
$$

It is important to note that $G_{0}^{\Lambda}$ depends on the mass $m$ only via rescaled temperature $\hat{\beta}=\beta m^{-1 / 2}$.

Remark 2.1 From (2.26)-(2.27) one gets that for $\Lambda \nearrow \mathbb{Z}^{\nu}$ the covariance $G_{0 ; j k}^{\Lambda}\left(\tau-\tau^{\prime}\right)$ converges pointwise to a translation invariant function $G_{0 ;|j-k|}\left(\tau-\tau^{\prime}\right)$ which is also a bounded continuous function and in fact it is the kernel of a positive self-adjoint operator in $L_{2}\left(\mathbb{Z}^{\nu} \otimes S_{\hat{\beta}} ; \mathbb{R}^{d}\right)$. From this convergence it follows that the corresponding process(measure) converges weakly to a limit process, which is a homogeneous Gaussian process on $\mathbb{Z}^{\nu}$ and periodic on $S_{\hat{\beta}}$ with values in $\mathbb{R}^{d}$.

By virtue of (2.20) and (2.23) it is clear that

$$
Z_{\Lambda}=\operatorname{Tr}_{\Lambda} e^{-\hat{\beta} \hat{H}_{\Lambda}}=Z_{\Lambda}^{0} \int_{\mathcal{H}_{\hat{\beta}, \Lambda}} d \mu_{0}^{\Lambda}(\phi) e^{-\int_{0}^{\hat{\beta}} d \tau \hat{V}\left(\phi_{\Lambda}(\tau)\right)}
$$


Now we can follow the line of reasoning of $[2,7,32]$. For every bounded function $\mathcal{A}\left(x_{\Lambda}\right)$ on $\mathbb{R}^{d|\Lambda|}$ we consider a bounded operator $A_{0}$ on $\mathcal{H}_{\Lambda}$ defined as multiplication on a bounded function:

$$
\left(A_{0} f\right)\left(x_{\Lambda}\right)=\mathcal{A}\left(x_{\Lambda}\right) f\left(x_{\Lambda}\right),
$$

and for any $t>0$ we consider the operator

$$
A_{t}=e^{-t \hat{H}_{\Lambda}} A_{0} e^{t \hat{H}_{\Lambda}} .
$$

Then for every set of bounded functions $\mathcal{A}^{(0)}, \ldots, \mathcal{A}^{(n)}$ and for increasing sequence of the "time" moments $0=t_{0} \leq t_{1} \leq \ldots \leq t_{n} \leq t_{n+1}=\hat{\beta}$, we define the operator

$$
A=\prod_{l=0}^{n} A_{t_{l}}^{(l)}=A_{0}^{(0)} e^{-t_{1} \hat{H}_{\Lambda}} A_{0}^{(1)} e^{-\left(t_{2}-t_{1}\right) \hat{H}_{\Lambda}} \ldots e^{-\left(t_{n}-t_{n-1}\right) \hat{H}_{\Lambda}} A_{0}^{(n)} e^{t_{n} \hat{H}_{\Lambda}}
$$

Then by (2.12) one gets:

$\hat{\rho}_{\Lambda}^{\hat{\beta}}(A)=\frac{1}{Z_{\hat{\beta}}(\Lambda)} \operatorname{Tr}\left(A_{0}^{(0)} e^{-t_{1} \hat{H}_{\Lambda}} A_{0}^{(1)} e^{-\left(t_{2}-t_{1}\right) \hat{H}_{\Lambda}} \ldots e^{-\left(t_{n}-t_{n-1}\right) \hat{H}_{\Lambda}} A_{0}^{(n)} e^{-\left(\beta-t_{n}\right) \hat{H}_{\Lambda}}\right)$.

This definition is correct because each operator $A_{0}^{(k)} e^{-\left(t_{k+1}-t_{k}\right) \hat{H}_{\Lambda}}, k=0,1, \ldots, n$, with $t_{0}=0$ and $t_{n+1}=\hat{\beta}$, is of the trace class. The state (2.32) is obviously analytic in the domain

$$
0<\operatorname{Re} t_{1}<\operatorname{Re} t_{2}<\ldots<\operatorname{Re} t_{n}<\hat{\beta}
$$

with boundary values at $\operatorname{Re} t_{i}=0, i=0, \ldots, n$, and it is uniformly bounded by the generalized Ginibre-Gruber inequality [23]:

$$
\operatorname{Tr}\left(\prod_{l=0}^{n} \alpha_{I m \tau_{l}}^{\Lambda}\left(A_{0}^{(l)}\right) e^{-\hat{\beta} \hat{H}_{\Lambda}}\right) \leq\left\|\prod_{l=0}^{n} A_{0}^{(l)}\right\| \operatorname{Tr}\left(e^{-\hat{\beta} \hat{H}_{L}}\right) .
$$

Here $\alpha_{\tau}^{\Lambda}(B)$ is the $C^{*}$-automorphism of $\mathcal{L}\left(\mathcal{H}_{\Lambda}\right)$ :

$$
\alpha_{\tau}^{\Lambda}(B)=e^{-i \tau \hat{H}_{\Lambda}} B e^{i \tau \hat{H}_{\Lambda}}, \quad B \in \mathcal{L}\left(\mathcal{H}_{\Lambda}\right)
$$


and so the boundary value of the state $\hat{\rho}_{\Lambda}^{\hat{\beta}}$ satisfies the KMS conditions (see $[2,32])$. Moreover, the following formula is true:

$$
\hat{\rho}_{\Lambda}^{\hat{\beta}}\left(\prod_{l=0}^{n} A_{t_{l}}^{(l)}\right)=\int_{\mathcal{H}_{\hat{\beta}, \Lambda}} \prod_{l=0}^{n} \mathcal{A}^{(l)}\left(\phi_{\Lambda}\left(t_{l}\right)\right) d \mu^{\Lambda}(\phi):=\left\langle\prod_{l=0}^{n} \mathcal{A}^{(l)}(\cdot)\right\rangle_{\mu^{\Lambda}},
$$

where $d \mu^{\Lambda}$ is the Gibbsian modification of the measure $d \mu_{0}^{\Lambda}$ :

$$
d \mu^{\Lambda}\left(\phi_{\Lambda}\right)=Z_{\hat{\beta}}(\Lambda)^{-1} e^{-\int_{0}^{\hat{\beta}} d \tau \hat{V}\left(\phi_{\Lambda}(\tau)\right)} d \mu_{0}^{\Lambda}\left(\phi_{\Lambda}\right)
$$

Following [7] we shall call this measure the Euclidean Gibbs Measure (EGM), which corresponds to our particular model (2.1)-(2.3) in this context.

So, due to this construction the Theorem 2.1 for quantum Gibbs states (2.32)-(2.33) can be reformulated as follows:

Theorem 2.2 For the system of quantum particles with Hamiltonian (2.1)(2.3) there exists a sufficiently small mass $m_{*}$ such that for any $0<m<m_{*}$ the weak limit of measures (2.34)

$$
\lim _{\Lambda \nearrow \mathbb{Z}^{\nu}} \mu^{\Lambda}=\mu
$$

exists and $\mu$ is a unique translation-invariant Gibbs measure on $\left(\Omega_{\hat{\beta}, \mathbb{Z}^{\nu}}\right.$, $\left.\Sigma_{\hat{\beta}, \mathbb{Z}^{\nu}}\right)$ in the set of tempered Gibbs measures, for all temperatures $T \geq 0$.

Remark 2.2 To prove the existence of the limit measure we are going to apply a cluster expansions procedure to the perturbed measure $\mu^{\Lambda}$ (2.34), which is constructed by the Hamiltonian (2.1)-(2.3) with p.b.c.(2.4). But, in fact, one can prove the convergence (in the thermodynamic limit) of cluster expansions for some non-empty set of boundary configurations of so-called tempered type (see (3.34) of the next Section), and so to prove the existence of the limit measure in the class of measures supported on these tempered configurations (the set of tempered Gibbs measures).

To prove the uniqueness of the limit measure in this set we use one elegant criterium of uniqueness due to [6] (Theorem 3.1). 
Remark 2.3 To prove the existence of the limit measure $\mu$ it is sufficient to prove the convergence of the averages (2.33) for local observables

$$
\mathcal{A}_{B}\left(\phi_{B}\right)=\prod_{j \in B} \mathcal{A}_{j}\left(\phi_{\tau_{j}}\right)
$$

for any bounded subsets $B \subset \Lambda$ and $\tau_{j} \in S_{\hat{\beta}}$.

Remark 2.4 Theorem 2.1 is equivalent to the Theorem 2.2 on the subalgebra constructed by (2.29)-(2.31). Then we use the fact that any operator $A \in \mathfrak{A}$ may be approximated by a linear combination of operators of the form (2.31) (see Theorem 2.2 of [32]). Hence the state $\hat{\rho}_{\Lambda}^{\hat{\beta}}(A)$ will also be approximated by (2.33).

\section{Cluster expansions and proof of main The- orems}

Remark 3.1 Before we construct the cluster expansions, which we need for the proof of Theorem 2.2 we recall the difference between the case $s>1$ in (1.3) and our case (1.5) with harmonic growth at infinity (see Section 1 and discussion in [43,44]). Notice that for harmonic interaction (i.e. for polynomials of the second degree) the rescaling (2.6) implies, for $s>1, a$ week interaction between oscillators proportional to $\mathrm{m}^{(s-1) /(s+1)}$. But for the harmonic growth of the one-site potential and for harmonic interaction the rescaling (2.6) leads to smallness of anharmonic perturbation $\hat{V}^{\Lambda}$, see (2.10).

Remark 3.2 Notice that the cluster expansions developed in [8,36,44] for $s>1$ are suited for small interaction between oscillators. In contrast to that the cluster expansions presented in the present paper are relevant for small one-site anharmonism and harmonic oscillators interaction. Therefore, it is suitable to arrange our reference measure in such a way it includes this interaction. Following the paper [2] it gives us the Gaussian measure representation for the state.

The cluster expansions technique for the Gaussian integrals with nondiagonal covariance was developed in [29] (see also $[30,49]$ ). Then it was 
applied in $[20,22]$ for the proof of the Debye screening in dilute charged particle systems. This type of cluster expansions includes derivatives acting on the Gibbs factor $\exp \left[-\hat{V}^{\Lambda}\right]$. Since the coefficient $b_{m}$ is proportional to $m^{1 / 2}$, and the coefficient $\delta_{m}$ is proportional to $m^{-1 / 2}$ (see $(2.10)$ ), we have no analyticity at $m=0$. Therefore, the estimates are delicate in the small-mass domain.

To prove the convergence of the measure $d \mu^{\Lambda}$ in the thermodynamic limit it is sufficient to prove the existence of limit state for observables $\mathcal{A}_{B}(2.35)$.

We would like to notice that similar to non-polynomial quantum field theory (see e.g. $[30,49]$ ) our cluster expansions correspond to a one-site perturbation, and not to the interaction between sites. To start the construction of cluster expansions we define new configuration space. First we consider $S_{\hat{\beta}}$ for large $\hat{\beta}$ including $\hat{\beta}=\infty$. So for a fixed $m$ we consider sufficiently low temperature such that critical behavior (if any) might be in the interval $[0, \hat{\beta}]$. Then just for convenience we consider $\hat{\beta} \in \mathbb{N}$ to define the partition of the interval $[0, \hat{\beta}]$ into unit intervals. So, we define

$$
\bar{\Delta}_{\hat{\beta}}:=\left\{\Delta_{\hat{\tau}}:=[\hat{\tau}, \hat{\tau}+1] \mid \hat{\tau}=0,1, \ldots, \hat{\beta}-1, \quad \hat{\beta} \in \mathbb{N}\right\},
$$

Then we define

$$
t:=(j, \tau), \quad j \in \mathbb{Z}^{\nu}, \quad \tau \in S_{\hat{\beta}},
$$

and the box

$$
T:=\Lambda \otimes S_{\hat{\beta}} \subset \mathbb{Z}^{\nu} \otimes \mathbb{R}_{+},
$$

where $\Lambda$ and $S_{\hat{\beta}}$ are defined in the previous section. With these notations we introduce

$$
G_{0}^{T}\left(t, t^{\prime}\right):=G_{0 ; j j^{\prime}}^{\Lambda}\left(\tau, \tau^{\prime}\right)
$$

For any $j \in \mathbb{Z}^{\nu}$ and $\Delta_{\hat{\tau}} \in \bar{\Delta}_{\hat{\beta}}$ we put

$$
\Delta_{j, \hat{\tau}}:=\left(j, \Delta_{\hat{\tau}}\right)
$$

and consider $T$ as finite union of "rods" $\Delta_{j, \hat{\tau}}, j \in \mathbb{Z}^{\nu}, \quad \hat{\tau}=0,1, \ldots, \hat{\beta}-1$. 
To construct the cluster expansions for the expectations of (2.35) we define (following [20]) a family of sets by the inductive procedure. Let for $n=1$

$$
X_{1}=Y_{1}=\left(B, \Delta_{B}\right), \quad \Delta_{B}=\bigcup_{j \in B} \Delta_{j},
$$

where $\Delta_{j}$ coincides with $\Delta_{\hat{\tau}}$ such that $\tau_{j} \in \Delta_{\hat{\tau}}$. For $n \geq 2$ we put

$$
Y_{n}=\left(j, \Delta_{\hat{\tau}}\right), j \in \Lambda \backslash B, \quad \text { or } \quad \Delta_{\hat{\tau}} \in \bar{\Delta}_{\hat{\beta}} \backslash \Delta_{B},
$$

and define

$$
X_{n}:=X_{n-1} \bigcup Y_{n}, \quad X_{n}^{c}:=T \backslash X_{n} .
$$

So, $Y_{n}$ for $n \geq 2$ are "rods" $\Delta_{j, \hat{\tau}}$, and $X_{n}$ are their unions.

Now define the sequence of new covariances (for $0 \leq s_{i} \leq 1, i=1, \ldots, n$ ):

$$
G_{0}^{T}\left(t, t^{\prime} ;(s)_{n}\right)=G_{0}^{T}\left(t, t^{\prime}\right) p\left(t-t^{\prime} ;(s)_{n}\right),
$$

where

$$
\begin{gathered}
p\left(t, t^{\prime} ;(s)_{n}\right)=\sum_{m=1}^{n+1} \mathbb{1}_{Y_{m}}(t) \mathbb{1}_{Y_{m}}\left(t^{\prime}\right)+ \\
\sum_{1 \leq l<m \leq n+1} s_{l} s_{l+1} \cdots s_{m-1}\left[\mathbb{1}_{Y_{l}}(t) \mathbb{1}_{Y_{m}}\left(t^{\prime}\right)+\mathbb{1}_{Y_{m}}(t) \mathbb{1}_{Y_{l}}\left(t^{\prime}\right)\right] .
\end{gathered}
$$

Here $\mathbb{1}_{Y}(t)$ is indicator of the "rod" $Y$, and the "rod" $Y_{n+1}$ we identify with $X_{n}^{c}$.

One can treat $G_{0}^{T}\left(t, t^{\prime} ;(s)_{n}\right)$ as a kind of "interaction potential" in the space of "cluster" configurations $\Lambda \times \bar{\Delta}_{\hat{\beta}}$. Then the interpolation parameters $s_{i}$ specify the intensity of this "interaction" between "rods" of $X_{i}$ and of $X_{i}^{c}$. Putting $s_{n}=0$ we get that there is no "interaction" between the "rods" of $X_{n}$ and "rods" of $X_{n}^{c}$. Then let $\mu_{0 ;(s)_{n}}^{T}$ be the Gaussian measure with covariance $G_{0}^{T}\left(t, t^{\prime} ;(s)_{n}\right)$. This is the kernel of a positive-definite operator in $L_{2}\left(T ; \mathbb{R}^{d}\right)$ because it is a linear combination with positive coefficients of operators of the form $\mathbb{1}_{X} G_{0}^{T} \mathbb{1}_{X}$, where $X$ is some of the unions of $Y_{m}$, see [21], [30, Sec.18.2] 
and Section 4 for details. It is clear from (3.10) that $\mu_{0 ;(1)_{n}}^{T}=\mu_{0}^{\Lambda}$. For $s_{n}=0$ one gets that the measure $d \mu_{0 ;(s)_{n-1}, 0}^{T}$ becomes the product measure

$$
d \mu_{0 ;(s)_{n-1}, 0}^{T}=d \mu_{0 ;(s)_{n-1}}^{\Lambda, X_{n}} d \mu_{0 ; 0}^{\Lambda, X_{n}^{c}}
$$

where the covariance of $d \mu_{0 ;(s)_{n-1}}^{\Lambda, X_{n}}$ is

$$
G_{0}^{\Lambda, X_{n}}\left(t, t^{\prime} ;(s)_{n-1}, 0\right)=G_{0}^{T}\left(t, t^{\prime} ;(s)_{n-1}, 0\right)-\mathbb{1}_{X_{n}^{c}}(t) G_{0}^{T}\left(t, t^{\prime}\right) \mathbb{1}_{X_{n}^{c}}\left(t^{\prime}\right)
$$

and the covariance of $d \mu_{0 ; 0}^{\Lambda, X_{n}^{c}}$ is

$$
G_{0}^{\Lambda, X_{n}}\left(t, t^{\prime}\right)=\mathbb{1}_{X_{n}^{c}}(t) G_{0}^{T}\left(t, t^{\prime}\right) \mathbb{1}_{X_{n}^{c}}\left(t^{\prime}\right) .
$$

Notice, also, that $\left.(s)_{n-1}\right|_{n=1}=\emptyset$ and $\left.d \mu_{0 ;(s)}^{\Lambda, X_{n}}\right|_{n=1}=: d \mu_{0}^{\Lambda, X_{1}}$, and that each step in the expansion can be obtained by applying the Newton-Leibnitz formula:

$$
\begin{gathered}
\left\langle\mathcal{A}_{B}(\cdot)\right\rangle_{\mu^{\Lambda}}=Z_{T}^{-1} \int_{\mathcal{H}_{\hat{\beta}, \Lambda}} d \mu_{0 ; 0}^{T}(\phi) \mathcal{A}_{B}\left(\phi_{B}\right) e^{-\hat{V}\left(\phi_{T}\right)}+ \\
Z_{T}^{-1} \int_{0}^{1} d s_{1} \frac{d}{d s_{1}} \int_{\mathcal{H}_{\hat{\beta}, \Lambda}} d \mu_{0 ; s_{1}}^{T}(\phi) \mathcal{A}_{B}\left(\phi_{B}\right) e^{-\hat{V}\left(\phi_{T}\right)}= \\
\int_{\mathcal{H}_{\hat{\beta}, \Lambda}} d \mu_{0}^{\Lambda, X_{1}}\left(\phi_{X_{1}}\right) \mathcal{A}_{B}\left(\phi_{B}\right) e^{-\hat{V}\left(\phi_{X_{1}}\right)} \frac{Z_{T}\left(X_{1}^{c}\right)}{Z_{T}}+R_{1}^{\Lambda},
\end{gathered}
$$

where $Z_{T}:=Z_{\hat{\beta}}(\Lambda)$ and

$$
Z_{T}(X)=\int_{\mathcal{H}_{\hat{\beta}, \Lambda}} d \mu_{0}^{T}(\phi) e^{-\hat{V}\left(\phi_{X}\right)}
$$

Using integration by parts formula to calculate the derivative w.r.t. parameter $s_{1}$ (see, for example, [30, Sec.9.1]) we obtain 


$$
R_{1}^{\Lambda}=Z_{T}^{-1} \int_{0}^{1} d s_{1} \int_{\mathcal{H}_{\hat{\beta}, \Lambda}} d \mu_{0 ; s_{1}}^{T}(\phi) \Delta_{\phi} \mathcal{A}_{B}\left(\phi_{B}\right) e^{-\hat{V}\left(\phi_{T}\right)}
$$

where

$$
\begin{gathered}
\Delta_{\phi}=\sum_{\alpha=1}^{d} \Delta_{\phi}^{(\alpha)} \\
\Delta_{\phi}^{(\alpha)}=\frac{1}{2} \sum_{j, k \in \Lambda} \int_{0}^{\hat{\beta}} d \tau \int_{0}^{\hat{\beta}} d \tau^{\prime} G_{0 ; j k}^{\Lambda}\left(\tau-\tau^{\prime}\right) \frac{d}{d s_{1}} p\left((j, \tau),\left(k, \tau^{\prime}\right) ; s_{1}\right) \frac{\delta^{2}}{\delta \phi_{j}^{(\alpha)}(\tau) \delta \phi_{k}^{(\alpha)}\left(\tau^{\prime}\right)} \\
=\frac{1}{2} \int_{T} d t \int_{T} d t^{\prime} G_{0}^{T}\left(t, t^{\prime}\right) \frac{d}{d s_{1}} p\left(t, t^{\prime} ; s_{1}\right) \frac{\delta^{2}}{\delta \phi^{(\alpha)}(t) \delta \phi^{(\alpha)}\left(t^{\prime}\right)} \\
=\int_{Y_{1}} d t \int_{X_{1}^{c}} d t^{\prime} G_{0}^{T}\left(t, t^{\prime}\right)\left(\frac{d}{d s_{1}} s_{1}\right) \frac{\delta^{2}}{\delta \phi^{(\alpha)}(t) \delta \phi^{(\alpha)}\left(t^{\prime}\right)} \\
=\sum_{Y_{2} \in X_{1}^{c}}\left(\frac{d}{d s_{1}} s_{1}\right) \Delta_{1,2}^{(\alpha)} .
\end{gathered}
$$

For definition of $\Delta_{p, p^{\prime}}^{(\alpha)}\left(p, p^{\prime} \in \mathbb{I}\right)$ see (3.24). Then, taking into account that

$$
e^{-\hat{V}\left(\phi_{T}\right)}=e^{-\hat{V}\left(\phi_{X_{2}}\right)} e^{-\hat{V}\left(\phi_{X_{2}^{c}}\right)}
$$

with $X_{2}=X_{1} \cup Y_{2}=Y_{1} \cup Y_{2}$, and putting (3.17)-(3.19) in to (3.16) we repeat the procedure in each term of the sum over $Y_{2}$ to carry out the second step of expansion. After $n_{T}=\left|T \backslash X_{1}\right|$ steps all "rods" in $T$ will be exhausted and we get the identity:

$$
\left\langle\mathcal{A}_{B}(\cdot)\right\rangle_{\mu^{\Lambda}}=\sum_{n=1}^{n_{T}} \sum_{\bar{Y} \subset T} \mathcal{K}_{\bar{Y}}^{T}\left(\mathcal{A}_{B}\right) F_{n}^{T}(\bar{Y})
$$


where

$$
\begin{gathered}
\mathcal{K}_{\bar{Y}}^{T}\left(\mathcal{A}_{B}\right)=\int(d s)^{n-1} f\left(\eta ;(s)_{n-2}\right) I_{n}^{T}\left(\bar{Y}, \eta, \mathcal{A}_{B} ;(s)_{n-1}\right), \\
I_{n}^{T}\left(\bar{Y}, \eta, \mathcal{A}_{B} ;(s)_{n-1}\right)=\int_{\mathcal{H}_{\hat{\beta}, \Lambda}} d \mu_{0 ;(s)_{n-1}}^{\Lambda, X_{n}}\left(\phi_{X_{n}}\right) \Delta(\eta, \bar{Y}) \mathcal{A}_{B}\left(\phi_{B}\right) e^{-\hat{V}\left(\phi_{X_{n}}\right)},
\end{gathered}
$$

with

$$
\Delta(\eta, \bar{Y})=\prod_{l=2}^{n} \Delta_{\eta(l), l}, \quad \Delta_{p, p^{\prime}}=\sum_{\alpha=1}^{d} \Delta_{p, p^{\prime}}^{(\alpha)},
$$

where $\eta:=\left\{(\eta(l), l)_{l=2}^{n}\right\}$ is a sequence of couples with $\eta(l)<l$, which we use to construct the trees estimates according the standard procedure, see e.g. [21], or [42] Ch.II.4. Here

$$
\begin{gathered}
\Delta_{p, p^{\prime}}^{(\alpha)}=\int_{Y_{p}} d t \int_{Y_{p^{\prime}}} d t^{\prime} G_{0}^{T}\left(t, t^{\prime}\right) \frac{\delta^{2}}{\delta \phi^{(\alpha)}(t) \delta \phi^{(\alpha)}\left(t^{\prime}\right)}, \\
f\left(\eta ;(s)_{n-2}\right)=\prod_{2 \leq m \leq n}\left(s_{\eta(m)} s_{\eta(m)+1} \cdots s_{m-2}\right), \\
\eta(m) \leq m-2, \quad s=\left(s_{1}, \ldots, s_{n-1}\right), \quad 0 \leq s_{j} \leq 1 .
\end{gathered}
$$

The second sum in (3.20) is over all possible sets $\bar{Y}=\left\{Y_{2}, \ldots, Y_{n}\right\}$ in $T$. The product in (3.25) is equal to 1 if $\eta(m)=m-1$. Finally

$$
F_{n}^{T}(\bar{Y})=\frac{Z_{T}\left(X_{n}^{c}\right)}{Z_{T}}, \quad Z_{T}\left(X_{n}^{c}\right)=Z_{T}\left(T \backslash X_{n}\right) .
$$

(For more details about construction of this type of expansions see, for example the survey [49].)

To prove Theorem 2.2 we use the representation (3.20) and the following collection of lemmas. 
Lemma 3.1 With the same assumptions as in Theorem 2.2 there exists a sufficiently small value of the mass $m_{*}$, and constant $C_{B}$ which does not depend on $T$ and on $m$, such that for all $0<m<m_{*}$

$$
\sum_{\bar{Y} \subset \mathbb{Z}^{\nu} \otimes S_{\hat{\beta}}} \mathcal{K}_{\bar{Y}}^{T}\left(\mathcal{A}_{B}\right) \leq C_{B} \varepsilon^{n-1},
$$

where $\varepsilon=\varepsilon(m) \rightarrow 0$ as $m \rightarrow 0$ and

$$
\lim _{\Lambda \nearrow \mathbb{Z}^{\nu}} \mathcal{K}_{\bar{Y}}^{T}\left(\mathcal{A}_{B}\right)=\mathcal{K}_{\bar{Y}}^{\beta}\left(\mathcal{A}_{B}\right) .
$$

Lemma 3.2 With the same assumptions as in Theorem 2.2 there exists a constant $c$ such that

$$
F_{n}^{T}(\bar{Y}) \leq e^{c(|B|+n-1)} .
$$

The constant $c$ does not depend on $\Lambda, \hat{\beta}, B, m$, and one has the limit:

$$
\lim _{\Lambda \nearrow \mathbb{Z}^{\nu}} F_{n}^{T}(\bar{Y})=F_{n}^{\hat{\beta}}(\bar{Y}) .
$$

We also need the following corollary of Lemmas 3.1 and 3.2.

Corollary 3.1 Let $B^{\prime} \subset \Lambda$ and $B \cap B^{\prime}=\emptyset$ and let the sum over $\bar{Y}$ be restricted so that $X_{n} \cap B \neq \emptyset$. Then

$$
\sum_{n=1}^{\infty} \sum_{\bar{Y}, X_{n} \cap B^{\prime} \neq \emptyset}\left|\mathcal{K}_{\bar{Y}}^{T}\left(\mathcal{A}_{B}\right)\right| F_{n}^{T}(\bar{Y}) \leq C_{B}^{\prime} e^{-\sqrt{a} \operatorname{dist}\left(B, B^{\prime}\right)},
$$

where $a$ is the constant of the one-site harmonic potential.

Now, using the standard "doubling measure" trick by J. Ginibre [28] (or [30, Sec.18.3]) we obtain from Corollary 3.1 the exponential clustering of correlations:

Lemma 3.3 With the same assumptions as in Theorem 2.2 and for any measurable functions $\mathcal{A}_{B_{1}}, \mathcal{A}_{B_{2}}$ w.r.t. $\sigma$-algebras $\Sigma_{\hat{\beta}, B_{1}}$ and $\Sigma_{\hat{\beta}, B_{2}}$ respectively, 
where $B_{1}, B_{2} \subset \Lambda$ and $B_{1} \cap B_{2}=\emptyset$, there exists a sufficiently small value of $m_{*}$, such that for $0<m<m_{*}$ and any temperature one has the estimate

$$
\begin{gathered}
\left\langle\mathcal{A}_{B_{1}}(\cdot) \mathcal{A}_{B_{2}}(\cdot)\right\rangle_{\mu^{\Lambda}}-\left\langle\mathcal{A}_{B_{1}}(\cdot)\right\rangle_{\mu^{\Lambda}}\left\langle\mathcal{A}_{B_{2}}(\cdot)\right\rangle_{\mu^{\Lambda}} \leq \\
\leq C_{1}\left(\mathcal{A}_{B_{1}}\right) C_{2}\left(\mathcal{A}_{B_{2}}\right) e^{-\sqrt{a} \operatorname{dist}\left(B_{1}, B_{2}\right)} .
\end{gathered}
$$

uniformly in $\Lambda$.

The proofs of Lemmas 3.1-3.2 and Corollary 3.1 are presented in the next Section. They imply the proof of our Theorem 2.2 for the case of p.b.c.

To prove the uniqueness of the limit measure in the Theorem 2.2 for non-periodic boundary conditions, we keep the DLR language (see [25]) and consider EGM, with some general boundary conditions $\xi$, from a class studied already in $[6,7]$. To this end we define the set of tempered configurations:

$$
\tilde{\Omega}_{\hat{\beta}}^{t}:=\bigcup_{\rho>0} \tilde{\Omega}_{\hat{\beta}}^{\rho}=\bigcup_{\rho>0}\left\{\xi \in \Omega_{\hat{\beta}, \mathbb{Z}^{\nu}} \mid \sum_{l \in \mathbb{Z}^{\nu}} e^{-\rho|l|}\left\|\xi_{l}\right\|_{L^{2}[0, \hat{\beta}]}<\infty\right\} .
$$

Remark 3.3 For tempered configurations $\Omega_{\hat{\beta}}^{t}$ defined in [6] one has: $\Omega_{\hat{\beta}}^{t} \subset$ $\tilde{\Omega}_{\hat{\beta}}^{t}$, since we include also configurations that may have an exponential growth at infinity.

First we define the harmonic measure with zero boundary conditions $\mu_{0}^{\Lambda}(\cdot \mid 0)$ in the same way as it was done in $(2.23)$ for $\mu_{0}^{\Lambda}(\cdot \mid p)$. Then for any $\xi \in \tilde{\Omega}_{\hat{\beta}}^{\rho}$ with $\rho<\sqrt{a}$, where $a$ is the one-site harmonic constant from (2.8), we define the perturbed measure with non-zero boundary conditions by

$$
d \mu^{\Lambda}(\phi \mid \xi):=Z_{\hat{\beta}}(\Lambda \mid \xi)^{-1} e^{-\int_{0}^{\hat{\beta}} d \tau \hat{V}\left(\phi_{\Lambda}(\tau)\right)+\frac{J}{2} \sum_{\left\langle l, l^{\prime}\right\rangle}^{\partial \Lambda} \int_{0}^{\hat{\beta}} d \tau \phi_{l}(\tau) \xi_{l^{\prime}}(\tau)} d \mu_{0}^{\Lambda}(\phi \mid 0)
$$

where 


$$
\sum_{\left\langle l, l^{\prime}\right\rangle}^{\partial \Lambda}(\ldots):=\sum_{l \in \Lambda, l^{\prime} \in \Lambda^{c},\left|l-l^{\prime}\right|=1}(\ldots) .
$$

We also need the following auxiliary measure $\tilde{\mu}^{\Lambda}\left(\cdot \mid y_{\Lambda}\right)$ on $\mathcal{H}_{\hat{\beta}, \Lambda}$, which depends on some fixed trajectories $y_{\Lambda} \in \Omega_{\hat{\beta}, \Lambda}$ :

$$
d \tilde{\mu}^{\Lambda}\left(x_{\Lambda} \mid y_{\Lambda}\right):=\tilde{Z}_{\hat{\beta}}\left(\Lambda \mid y_{\Lambda}\right)^{-1} e^{-\int_{0}^{\hat{\beta}} d \tau \tilde{V}\left(x_{\Lambda}(\tau) \mid y_{\Lambda}(\tau)\right)} d \mu_{0}^{\Lambda}\left(x_{\Lambda} \mid 0\right),
$$

where (see (2.10)):

$$
\tilde{V}\left(x_{\Lambda}(\tau) \mid y_{\Lambda}(\tau)\right)=b_{m} \sum_{j \in \Lambda}\left[e^{-\delta_{m}\left(x_{j}(\tau)+y_{j}(\tau)\right)^{2} / 4}+e^{-\delta_{m}\left(x_{j}(\tau)-y_{j}(\tau)\right)^{2} / 4}\right] .
$$

It is clear that with the measure $\tilde{\mu}^{\Lambda}\left(\cdot \mid y_{\Lambda}\right)$ one can construct the same cluster expansion as in (3.23), but with additional dependence on configurations $y_{\Lambda}$ :

$$
\left\langle\mathcal{A}_{B}(\cdot)\right\rangle_{\tilde{\mu}^{\Lambda}\left(\cdot \mid y_{\Lambda}\right)}=\sum_{n=1}^{n_{T}} \sum_{\bar{Y} \subset T} \mathcal{K}_{\bar{Y}}^{T}\left(\mathcal{A}_{B} \mid y_{\Lambda}\right) F_{n}^{T}\left(\bar{Y} \mid y_{\Lambda}\right),
$$

In the next Section we prove the following lemma:

Lemma 3.4 Let $m<m_{*}$ as in Lemmas 3.1-3.3. Then for the measure $\tilde{\mu}^{\Lambda}\left(\cdot \mid y_{\Lambda}\right)$, defined by (3.37)-(3.38), the estimates (3.30)-(3.33) are true with the same constants which do not depend on configurations $y_{\Lambda}$.

Proof of Theorem 2.2.

Existence. First we prove the existence of measures with p.b.c. and zero boundary conditions As we mentioned in Remark 2.1 the covariance $G_{0 ; j k}^{\Lambda}(\tau-$ $\tau^{\prime}$ ) converges pointwise to the translation invariant function $G_{0 ;|j-k|}\left(\tau-\tau^{\prime}\right)$ and the corresponding measures $d \mu_{0}$ and $d \mu_{0 ;(s)(n-1)}^{X_{n}}$ are defined on $\Sigma_{\hat{\beta}}$. So, 
using Lemmas 3.1 and 3.2 we can rewrite the expansion (3.20) in the thermodynamic limit:

$$
\left\langle\mathcal{A}_{B}(\cdot)\right\rangle_{\mu}=\sum_{n \geq 1} \sum_{\bar{Y} \subset \mathbb{Z}^{\nu} \otimes S_{\hat{\beta}}} \mathcal{K}_{\bar{Y}}^{\hat{\beta}}\left(\mathcal{A}_{B}\right) F_{n}^{\hat{\beta}}(\bar{Y}),
$$

with the same definitions of $I_{n}$ and $\Delta(\eta, \bar{y})$ (see (3.22), (3.23)), but with $G_{0}$ and $\mu_{0 ;(s)_{n-1}}^{X_{n}}$ instead of $G_{0}^{T}$ and $\mu_{0 ;(s)_{n-1}}^{\Lambda, X_{n}}$.

Hence, there exists probability measures $\mu_{B}$ indexed by $B \subset \mathbb{Z}^{\nu}$ such that

$$
\left\langle\mathcal{A}_{B}\right\rangle=\int_{\mathcal{H}_{\hat{\beta}, \Lambda}} \mathcal{A}_{B}(\phi) d \mu_{B}(\phi) .
$$

All these measures are consistent and consequently by the Kolmogorov Theorem one can construct a unique limit measure $\mu$ on the $\sigma$-algebra $\Sigma_{\hat{\beta}, \mathbb{Z}^{\nu}}$.

Remark 3.4 In the case of general boundary conditions $\xi \in \tilde{\Omega}_{\hat{\beta}}^{\rho}$ with $\rho<$ $\sqrt{a}$, the factors $\mathcal{K}_{\bar{Y}}^{\hat{\beta}}, F_{n}^{\hat{\beta}}$, and the constants $c, C_{B}$ in the Lemmas 3.1-3.2 will depend on $\xi$. This means that from convergence of the cluster expansions we can obtain only existence of the limit measure, but not uniqueness.

Uniqueness. To prove the uniqueness we use the following

Proposition 3.1 (see Theorem 3.1 of $[6]$ )

Suppose that for every $l_{0} \in \mathbb{Z}^{\nu}$ and $\tau_{0} \in[0, \hat{\beta}]$, for every increasing sequence of boxes $\Lambda_{n} \subset \mathbb{Z}^{\nu}\left(\Lambda_{n} \rightarrow \mathbb{Z}^{\nu}\right)$, such that $l_{0} \in \Lambda_{n}$ for all $n$, and for any two configurations $\xi, \eta \in \tilde{\Omega}_{\hat{\beta}}^{\rho}, \rho<\sqrt{a}$, one has the convergence:

$$
\left\langle\phi_{l_{0}}\left(\tau_{0}\right)\right\rangle_{\mu^{\Lambda_{n}(\cdot \mid \xi)}}-\left\langle\phi_{l_{0}}\left(\tau_{0}\right)\right\rangle_{\mu^{\Lambda_{n}(\cdot \mid \eta)}} \rightarrow 0
$$

as $n \rightarrow \infty$. Then there exist a unique limit measure $\mu$ which does not depend on configurations $\xi, \eta$.

This statement is a quantum version of some known previous results, namely, the monotonicity arguments [41] for the measures on the configurations in the Classical Statistical Mechanics and a priori estimates for EGM 
due to $[10,11]$. These arguments are true for the wide class of anharmonic potentials with ferromagnetic type of interaction. We use this statement as follows. Consider

$$
\begin{gathered}
\left\langle\phi_{l_{0}}\left(\tau_{0}\right)\right\rangle_{\mu^{\Lambda_{n}}(\cdot \mid \xi)}-\left\langle\phi_{l_{0}}\left(\tau_{0}\right)\right\rangle_{\mu^{\Lambda_{n}}(\cdot \mid \eta)}= \\
=\int_{0}^{1} d s \frac{d}{d s}\left\langle\phi_{l_{0}}\left(\tau_{0}\right)\right\rangle_{\mu^{\Lambda_{n}}(\cdot \mid \eta+s \zeta)}, \quad \zeta=\xi-\eta .
\end{gathered}
$$

After differentiation we obtain:

$$
\begin{gathered}
\left|\left\langle\phi_{l_{0}}\left(\tau_{0}\right)\right\rangle_{\mu^{\Lambda_{n}(\cdot \mid \xi)}}-\left\langle\phi_{l_{0}}\left(\tau_{0}\right)\right\rangle_{\mu^{\Lambda_{n}}(\cdot \mid \eta)}\right| \leq \\
\sup _{s \in[0,1]} \frac{J}{2} \sum_{\left\langle l, l^{\prime}\right\rangle}^{\partial \Lambda} \int_{0}^{\hat{\beta}} d \tau K_{l l_{0}}^{\Lambda}\left(\tau, \tau_{0} \mid \eta+s \zeta\right) \zeta_{l^{\prime}}(\tau),
\end{gathered}
$$

where

$$
K_{l l^{\prime}}^{\Lambda}\left(\tau, \tau^{\prime} \mid \eta\right)=\left\langle\phi_{l}(\tau) \phi_{l^{\prime}}\left(\tau^{\prime}\right)\right\rangle_{\mu^{\Lambda}(\cdot \mid \eta)}-\left\langle\phi_{l}(\tau)\right\rangle_{\mu^{\Lambda}(\cdot \mid \eta)}\left\langle\phi_{l^{\prime}}\left(\tau^{\prime}\right)\right\rangle_{\mu^{\Lambda}(\cdot \mid \eta)} .
$$

It is easy to see that the convergence (3.41) follows from the

Lemma 3.5 For $m<m_{*}$ there are constants $C_{i}, i=1,2$ independent of $\tau, \tau^{\prime}, \Lambda, \eta$, such that

$$
K_{l l^{\prime}}^{\Lambda}(i) \leq C_{i} e^{-\sqrt{a}\left|l-l^{\prime}\right|}
$$

where

$$
K_{l l^{\prime}}^{\Lambda}(1)=K_{l l^{\prime}}^{\Lambda}\left(\tau, \tau^{\prime} \mid \eta\right), \quad \text { and } \quad K_{l l^{\prime}}^{\Lambda}(2)=\int_{0}^{\hat{\beta}} d \tau^{\prime} K_{l l^{\prime}}^{\Lambda}\left(\tau, \tau^{\prime} \mid \eta\right)
$$


Finally, applying (similar to [6]) the Cauchy-Schwarz inequality in (3.43) and using (3.45), we get (3.41).

\section{Proof of Theorem 2.1.}

As we mentioned in Section 2, our Theorem 2.2 is, in fact, a reformulation of Theorem 2.1 on the algebra which is constructed by (2.29)-(2.33). The uniqueness of the limit Gibbs state on the corresponding algebra follows from (3.43)-(3.46) by virtue of Proposition 3.1 for the $\left(\Omega_{\hat{\beta}, \mathbb{Z}^{\nu}}, \Sigma_{\hat{\beta}, \mathbb{Z}^{\nu}}\right)$ on the set of tempered configurations (3.34). The convergence of cluster expansions gives also analyticity of the limit state in the circle $\left|m-m_{*} / 2\right|<m_{*} / 2$ of the complex plane $m$. The end of the proof is based on the same arguments as in Theorem 4.1 of [2].

\section{Convergence of the cluster expansions}

To prove the convergence of the cluster expansions (3.20) for $\Lambda \nearrow \mathbb{Z}^{\nu}$ we should first give demonstrations of Lemmas 3.1 and 3.2.

Proof of Lemma 3.1. To simplify the proof we omit indexes $\alpha=1, \ldots, d$ and we take into account that the number of derivatives for each order of $n$ should increase as $d^{n-1}$. To write down the general term for any fixed tree $\eta$ (see $(3.23)$ ) let us introduce (following $[16,17]$ ) the numbers $d_{\eta}(k), k=1, \ldots, n$ which are the characteristics of tree $\eta$, i.e. $d_{\eta}(k)$ is a number of lines, coming into the vertex $k$ from vertices $m(m>k)$. It is clear that $n_{1}=d_{\eta}(1)$, $n_{k}=d_{\eta}(k)+1$ for $k=2, \ldots, n$, are exactly the number of lines coming from vertices $k=1, \ldots, n$ and these numbers coincides with the numbers of derivatives supported in $Y_{1}, Y_{2}, \ldots, Y_{n}$. Hence,

$$
\sum_{k=1}^{n} d_{\eta}(k)=n-1 \quad \text { and } \quad \sum_{k=1}^{n} n_{k}=2(n-1) .
$$

Let us rewrite the operator $\Delta(\eta, \bar{Y})$ in the following form

$$
\begin{gathered}
\Delta(\eta, \bar{Y})=\left(\prod_{k=1}^{n} \prod_{l=1}^{n_{k}} \int_{Y_{k}} d t_{k}^{(l)}\right) \prod_{k=2}^{n} G_{0}^{T}\left(t_{\eta(k)}^{\left(\eta_{k}\right)}-t_{k}^{\left(n_{k}\right)}\right) \times \\
\prod_{k=1}^{n} \frac{\delta^{n_{k}}}{\delta \phi\left(t_{k}^{(1)}\right) \cdots \delta \phi\left(t_{k}^{\left(n_{k}\right)}\right)} .
\end{gathered}
$$


Here we put

$$
\eta_{2}:=1, \quad \eta_{k}:=1+\sum_{l=2}^{k-1} \delta_{\eta(l), \eta(k)}, \quad k \geq 3,
$$

where $\delta_{\eta(l), \eta(k)}$ is the symbol of Kronecker. In the following we also put $t_{k}^{\left(n_{k}\right)}:=t_{k}$.

The main technical estimate is the following

Proposition 4.1 Let $D_{n_{k}}$ be parts of operator $\Delta(\eta, \bar{Y})$ and factor $e^{-\hat{V}\left(\phi_{X_{n}}\right)}$ corresponding to the $k$-th vertex on the tree $\eta$ :

$$
\begin{gathered}
D_{n_{k}}=D_{n_{k}}\left(t_{\eta(k)}^{\left(\eta_{k}\right)} ; t_{1}, \ldots, t_{d_{\eta}(k)}\right)= \\
=\int_{\left(Y_{k}\right)^{n_{k}}} G_{0}^{T}\left(t_{\eta(k)}^{\left(\eta_{k}\right)}-t_{k}^{\left(n_{k}\right)}\right) G_{0}^{T}\left(t_{k}^{(1)}-t_{1}\right) \cdots G_{0}^{T}\left(t_{k}^{\left(d_{\eta}(k)\right)}-t_{d_{\eta}(k)}\right) \times \\
\times \frac{\delta^{n_{k}}}{\delta \phi\left(t_{k}^{(1)}\right) \cdots \delta \phi\left(t_{k}^{\left(n_{k}\right)}\right)} e^{-\hat{V}\left(\phi_{Y_{k}}\right)} .
\end{gathered}
$$

Then for $b_{m}<1$ the following estimate is true:

$$
\begin{gathered}
D_{n_{k}}\left(t_{\eta(k)}^{\left(\eta_{k}\right)} ; t_{1}, \ldots, t_{d_{\eta}(k)}\right) \leq 2^{n_{k}} b_{m} \delta_{m}^{n_{k} / 2} n_{k} ! \times \\
\times \sup _{(*)_{d_{\eta}(k)}} \int_{Y_{k}} d t_{k} G_{0}^{T}\left(t_{\eta(k)}^{\left(\eta_{k}\right)}-t_{k}^{\left(n_{k}\right)}\right) G_{0}^{*, T}\left(\cdot-t_{1}\right) \cdots G_{0}^{*, T}\left(\cdot-t_{d_{\eta}(k)}\right) e^{-\delta_{m} \phi\left(t_{k}\right)^{2} / 4}
\end{gathered}
$$

here supremum is taken over all terms in the product, where one can choose $G_{0}^{*, T}\left(\cdot-t_{i}\right)$ equal either to $G_{0}^{T}\left(t_{k}-t_{i}\right)$, or $\int_{Y_{k}} d t^{\prime} G_{0}^{T}\left(t^{\prime}-t_{i}\right)$.

Proof. For simplicity we demonstrate this proposition for the case when instead of functional derivatives we have the ordinary derivatives, since exactly the same line of reasoning gives the proof for functional derivatives.

Let $X=-b_{m} e^{-\delta_{m} x^{2} / 2}$ denote the "scalar prototype" of our anharmonic potential in (4.3), supported in $Y_{k}$. Then it easy to estimate by induction the value of $n$-th derivatives of $X$ :

$$
\left|X^{(n)}\right| \leq b_{m} 2^{n} \delta_{m}^{n / 2} \sqrt{n !} e^{-\delta_{m} x^{2} / 4} .
$$


This inequality can be obtained by induction using the recursion relation:

$$
I_{n}(x)=-\delta_{m} x I_{n-1}(x)-(n-1) \delta_{m} I_{n-2}(x), \quad I_{n}(x)=e^{\delta_{m} x^{2} / 4} X^{(n)}(x),
$$

and the inequality:

$$
|x|^{k} e^{-\delta_{m} x^{2} / 4} \leq 2^{k / 2} \delta_{m}^{-k / 2} \sqrt{k !},
$$

Now using the Leibnitz formula for the product $X^{(1)} e^{X}$, where the first derivative $X^{(1)}=b_{m} \delta_{m} x e^{-\delta_{m} x^{2} / 2}$, and the estimate (4.5), again by induction one obtains:

$$
\left|\frac{d^{n}}{d x^{n}} e^{X}\right| \leq 2^{n} b_{m} \delta_{m}^{n / 2} n ! e^{-\delta_{m} x^{2} / 4}
$$

We also used here the fact that $e^{X} \leq 1$.

Remark 4.1 The inequality (4.7) explains the provenance of the coefficient in (4.4), since by the same way this coefficient appears when one passes to functional derivatives (as in (4.3)), instead of the ordinary ones.

Remark 4.2 Notice that we also should consider the terms in which some derivatives act on the factor $\mathcal{A}_{B}$. But, since $\mathcal{A}_{B}$ is supported only in $Y_{1}=$ $X_{1}$, then even in the case, when for a given tree $\eta$ one has all $\eta(k)=1$, for $k=2, \ldots, n$, and $(n-1)$ derivatives act only on $\mathcal{A}_{B}$, the other $(n-1)$ derivatives in $\Delta(\eta, \bar{Y})$ act on the factor $\exp \left[-\hat{V}_{a}\left(\phi_{X_{n}}\right)\right]$. Hence, we obtain the powers of the small parameter $b_{m}$, that we need.

This finishes the proof of Proposition.

As a result we can write for $I_{n}^{T}$ the following estimate:

$$
\begin{gathered}
I_{n}^{T}\left(\bar{Y}, \eta, \mathcal{A}_{B} ;(s)_{n-1}\right) \leq C\left(\mathcal{A}_{B}\right)\left(8 d b_{m} \delta_{m}\right)^{n-1} \prod_{k=1}^{n-1} d_{\eta}(k) ! \times \\
\times \prod_{k=2}^{n}\left(\sup _{t_{k}^{\prime}} \int_{Y_{k}} d t_{k} G_{0}^{T}\left(t_{k}^{\prime}, t_{k}\right)\right) \int_{\mathcal{H}_{\hat{\beta}, \Lambda}} d \mu_{0 ;(s)_{n-1}}^{\Lambda, X_{n}}\left(\phi_{X_{n}}\right) \prod_{k=1}^{n} e^{-\delta_{m} \phi\left(t_{k}\right)^{2} / 4}, \quad t_{k} \in Y_{k} .
\end{gathered}
$$

The next step is to estimate the Gaussian integral in (4.8). 


\section{Proposition 4.2 Let}

$$
\tilde{I}_{n}=\int_{\mathcal{H}_{\hat{\beta}, \Lambda}} d \mu_{0,(s)_{n-1}}^{\Lambda, X_{n}}\left(\phi_{X_{n}}\right) \prod_{k=1}^{n} e^{-\delta_{m} \phi\left(t_{k}\right)^{2} / 4}, \quad t_{k} \in Y_{k}, \quad n \geq 2,
$$

then there exists a constant $c$, which depends on parameters $d, J, a$ such that

$$
\tilde{I}_{n} \leq e^{c\left|X_{n}\right|}\left(\frac{2 \sqrt{a}}{2 \sqrt{a}+\delta_{m}}\right)^{d\left|X_{n}\right| / 4}
$$

Proof. Write the product in (4.9) as new a Gaussian integral w.r.t. the measure $d \gamma(z)=1 /(2 \pi)^{d n / 2} \exp \left[-z^{2} / 2\right], z \in \mathbb{R}^{d n}$ :

$$
\tilde{I}_{n}=\int_{\mathcal{H}_{\hat{\beta}, \Lambda}} d \mu_{0 ;(s)_{n-1}}^{\Lambda, X_{n}}\left(\phi_{X_{n}}\right) \int_{\mathbb{R}^{d n}} d \gamma(z) e^{i \sqrt{\delta_{m} / 2} \sum_{k=1}^{n} z_{k} \cdot \phi\left(t_{k}\right)}
$$

Interchanging integrals one obtains

$$
\tilde{I}_{n}=\int_{\mathbb{R}^{d n}} d \gamma(z) e^{-\delta_{m} / 4 \sum_{k, k^{\prime}=1}^{n} z_{k} z_{k^{\prime}} G_{0}^{\Lambda, X_{n}}\left(t_{k}, t_{k^{\prime}} ;(s)_{n-1}, 0\right)},
$$

where $G_{0}^{\Lambda, X_{n}}$ is defined by (3.12). By construction (3.9)-(3.10) the function $G_{0}^{\Lambda, X_{n}}\left(t_{k}, t_{k^{\prime}} ;(s)_{n-1}, 0\right)$ is a convex combination of "diagonalized" covariances (see [21], or [30, Sec.18.2]):

$$
G_{0}^{\Lambda, X_{n}}\left(t_{k}, t_{k^{\prime}} ;(s)_{n-1}, 0\right)=\sum_{i} \lambda_{i} G_{0}^{(i)}, \quad \sum_{i} \lambda_{i}=1,
$$

where coefficients $\lambda_{i}$ are monomials of the form $s_{k_{1}} \cdots s_{k_{p}}\left(1-s_{l_{1}}\right) \cdots\left(1-s_{l_{p^{\prime}}}\right)$ and

$$
G_{0}^{(i)}=\sum_{k} \mathbb{1}_{Z_{k}(i)} G_{0}^{T} \mathbb{1}_{Z_{k}(i)}, \quad \bigcup_{k} Z_{k}(i)=X_{n} \quad \text { for all } i .
$$


Substituting (4.13) into (4.12), applying then the Hölder inequality, and returning back to the measure $d \mu_{G_{0}^{\Lambda}}^{\Lambda, X_{n}}$, defined by the covariance (4.14), one gets:

$$
\begin{gathered}
\tilde{I}_{n} \leq \prod_{i}\left(\int_{\mathbb{R}^{d n}} d \gamma(z) e^{-\delta_{m} / 4 \sum_{k, k^{\prime}=1}^{n} z_{k} z_{k^{\prime}} G_{0}^{\Lambda, X_{n}}\left(t_{k}, t_{k^{\prime}}\right)}\right)^{\lambda_{i}}= \\
\prod_{i}\left(\int_{\mathcal{H}_{\hat{\beta}, \Lambda}} d \mu_{G_{0}^{\Lambda, X_{n}}}^{(i)}\left(\phi_{X_{n}}\right) \prod_{k=1}^{n} e^{-\delta_{m} \phi\left(t_{k}\right)^{2} / 4}\right)^{\lambda_{i}}=: \prod_{i} K_{i}^{\lambda_{i}} .
\end{gathered}
$$

By virtue of (4.14) we have:

$$
K_{i}=\prod_{k}\left(\int_{\mathcal{H}_{\hat{\beta}, \Lambda}} d \mu_{G_{0}^{\Lambda, Z}}^{(i)}\left(\phi_{X_{n}}\right) \prod_{Y \in Z_{k}(i)} e^{-\delta_{m} \phi\left(t_{Y}\right)^{2} / 4}\right)=: \prod_{k} J_{k}\left(Z_{k}(i)\right)
$$

By definition (2.23) one gets for $\Lambda=Z_{k}(i)$ and $F\left(\phi_{Z_{k}(i)}\right)=\prod_{Y \in Z_{k}(i)} e^{-\delta_{m} \phi\left(t_{Y}\right)^{2} / 4}$ :

$$
J_{k}\left(Z_{k}(i)\right)=C_{0}\left(Z_{k}(i) ; J\right)^{-1} \int_{\Omega_{\hat{\beta}, \Lambda}} d \omega_{Z_{k}(i)} e^{-\frac{J}{4} \int_{Z_{k}(i)}^{*} d t\left(\omega(t)-\omega\left(t_{*}\right)\right)^{2}} \prod_{Y \in Z_{k}(i)} e^{-\delta_{m} \omega_{Y}\left(\tau_{Y}\right)^{2} / 4}
$$

Here the measure $d \omega_{Z_{k}(i)}$ is defined by l.h.s. of (2.23), with $J=0$ in definition of operator $B_{\Lambda}(2.15)$ and with $\Lambda_{k}(i):=Z_{k}(i) \cap \Lambda, \quad S_{k}(i):=Z_{k}(i) \cap S_{\hat{\beta}}$ :

$$
\int_{\Omega_{\hat{\beta}, \Lambda}} d \omega_{Z_{k}(i)}(\ldots)=\int_{\mathbb{R}^{d|\Lambda|}} d x_{\Lambda} \int_{\Omega_{\hat{\beta}, \Lambda}} W_{x_{\Lambda} ; x_{\Lambda}}^{\hat{\beta}}\left(d \omega_{\Lambda}\right) e^{-\frac{a}{2} \int_{0}^{\hat{\beta}} d \tau\left(\omega_{\Lambda}(\tau), \omega_{\Lambda}(\tau)\right)+\frac{d}{2} \operatorname{Tr} B_{\Lambda}}(\ldots) .
$$

We put also :

$$
\int_{Z_{k}(i)}^{*} d t\left(\omega(t)-\omega\left(t_{*}\right)\right)^{2}:=\sum_{\left\langle l, l^{\prime}\right\rangle \subset \Lambda_{k}(i)} \int_{S_{k}(i)} d \tau\left(\omega_{l}(\tau)-\omega_{l^{\prime}}(\tau)\right)^{2}
$$


The normalization factor $C_{0}:=C_{0}\left(Z_{k}(i) ; J\right)$ is the integral (4.17) calculated for $\delta_{m}=0$. By the Cauchy-Schwarz inequality we get from integral (4.17) that

$$
J_{k}\left(Z_{k}(i)\right) \leq \frac{C_{0}\left(Z_{k}(i) ; 2 J\right)^{1 / 2}}{C_{0}\left(Z_{k}(i) ; J\right)}\left(\prod_{Y \in Z_{k}(i)} \int_{\Omega_{\hat{\beta}}} d \omega_{Y} e^{-\delta_{m} \omega_{Y}\left(\tau_{Y}\right)^{2} / 2}\right)^{1 / 2}
$$

The factors $C_{0}$ for $J$ and $2 J$ can be calculated explicitly using (2.24), (2.18). The integral in the product (4.19) can be estimated (for $|Y|=1$ ) by

$$
\begin{gathered}
\int_{\Omega_{\hat{\beta}}} d \omega_{Y} e^{-\delta_{m} \omega\left(t_{Y}\right)^{2} / 2}=\left.C_{0}(Y, 0) \int_{\mathcal{H}_{\hat{\beta}, \Lambda}} d \mu_{G_{0}^{\Lambda}}^{Y}\left(\phi_{Y}\right) e^{-\delta_{m} \phi_{l_{Y} / 2}\left(\tau_{Y}\right)^{2}}\right|_{J=0}= \\
C_{0}(Y, 0)\left(1+\frac{\delta_{m}}{2 \sqrt{a}} \cdot \frac{e^{\hat{\beta} \sqrt{a}}+1}{e^{\hat{\beta} \sqrt{a}}-1}\right)^{-d / 2} \leq e^{\mathrm{const}|Y|}\left(\frac{2 \sqrt{a}}{2 \sqrt{a}+\delta_{m}}\right)^{d / 2} \cdot
\end{gathered}
$$

This finishes the proof of the Proposition.

Now we return to the proof of Lemma 3.1. Since

$$
\begin{gathered}
\sum_{Y} \int_{Y} d t G_{0}^{T}\left(t^{\prime}-t\right)=\int_{T} d t G_{0}^{T}\left(t^{\prime}-t\right)=\sum_{j \in \Lambda} \int_{0}^{\hat{\beta}} d \tau G_{0, i j}^{\Lambda}\left(\tau^{\prime}-\tau\right)= \\
=: \sum_{j \in \Lambda} D_{0}^{\Lambda}(i-j) \leq \sum_{j \in \mathbb{Z}^{\nu}} D_{0}(j)=: C_{G},
\end{gathered}
$$

then taking into account dependence of $b_{m}$ and $\delta_{m}$ on $m$ (see (2.10)) and definition (3.21), we obtain by summing over trees $\eta$ the estimate:

$$
\begin{gathered}
\left|\mathcal{K}_{\bar{y}}^{T}\left(\mathcal{A}_{B}\right)\right| \leq C^{\prime}\left(\mathcal{A}_{B}\right)\left(16 b \sqrt{a} C_{G} m^{d / 8}\right)^{n-1} \times \\
\times \sum_{\eta} \prod_{p=1}^{n} d_{\eta}(p) ! \int_{0}^{1}(d s)^{n-1} f\left(\eta ;(s)_{n-1}\right) .
\end{gathered}
$$


The last step of the proof is due to the Battle-Federbush inequality [16,17]:

$$
\sum_{\eta} \prod_{p=1}^{n} d_{\eta}(p) ! \int_{0}^{1}(d s)^{n-1} f\left(\eta ;(s)_{n-2}\right) \leq 4^{n}, \quad n=|\eta| .
$$

This yields (3.28) with

$$
\varepsilon(m)=64 b \sqrt{a} C_{G} m^{d / 8}
$$

Now taking into account (3.30), we obtain the value of the small-mass threshold $m_{*}$ :

$$
m_{*}=\left(64 b \sqrt{a} C_{G} e^{c}\right)^{-8 / d} .
$$

Proof of Lemma 3.2. The proof is standard. One can use general theory of polymer-type expansions [42] (or, equivalently, the Kirkwood-Salsburg type technique [30, Sec.18.5]), see, also, [49].

Proof of Corollary 3.1. If we restrict the sum in the cluster expansion (3.20)-(3.27) in such a way that, for example, $Y_{2} \subset B^{\prime}$, then all terms in this expansion are proportional to $G_{0}^{T}\left(t_{1}, t_{2}\right)$, where $t_{1} \in B, t_{2} \in B^{\prime}$, then (3.32) follows from the asymptotic behaviour of $G_{0,|i-j|}(\tau)$ in the variable $|i-j|$. Note, also, that in the case of p.b.c. (see e.g.[30])

$$
G_{0 ;|i-j|}(\tau \mid p) \leq G_{0 ;|i-j|}(\tau \mid 0),
$$

and it has exponential decay of $G_{0 ;|i-j|}(\tau \mid 0)$ in $|i-j|$, which is uniform in $\tau$.

Proof of Lemma 3.4. To prove this lemma one has to check that Propositions 4.1 and 4.2 are true for the auxiliary potential (3.38). It is easy to verify that (4.5)-(4.7) are true for $X_{ \pm}=X_{ \pm}(x \mid y)=-b_{m} e^{-\delta_{m}(x \pm y)^{2} / 4}$ with $\delta_{m} / 2$ instead of $\delta_{m}$. So, the estimate $(4.4)$ is the same but with $\left[e^{-\delta_{m}\left(x\left(t_{k}\right)+y\left(t_{k}\right)\right)^{2} / 8}+\right.$ $\left.e^{-\delta_{m}\left(x\left(t_{k}\right)-y\left(t_{k}\right)\right)^{2} / 8}\right]$ instead of $e^{-\delta_{m} \phi\left(t_{k}\right)^{2} / 4}$. Then in the proof of Proposition 4.2 we have to modify the integral (4.20) to

$$
\left.\int_{\mathcal{H}_{\hat{\beta}, \Lambda}} d \mu_{G_{0}^{\Lambda}}^{Y}\left(x_{Y}\right) e^{-\frac{\delta m}{8}\left(x_{l_{Y}}\left(\tau_{Y}\right) \pm y_{Y}\left(\tau_{Y}\right)\right)^{2}}\right|_{J=0}
$$


which gives

$$
\left.C_{0}(Y, 0)\left(1+\frac{\delta_{m}}{4} G_{0}^{\Lambda}(0)\right)^{-d / 2} e^{-\frac{\delta_{m} y^{2}}{8\left(1+\delta_{m} G_{0}^{\Lambda}(0) / 4\right)}}\right|_{J=0}
$$

Since the last exponent is less than one, the dependence on the configuration $y$ disappears, and this finishes the proof.

Proof of Lemma 3.5. We follow the line of reasoning in the proof of Theorem $3.2[6]$. Let $d \tilde{\mu}_{0}^{\Lambda}\left(\phi, \phi^{\prime} \mid 0\right):=d \mu_{0}^{\Lambda}(\phi \mid 0) \otimes d \mu_{0}^{\Lambda}\left(\phi^{\prime} \mid 0\right)$. Then we can rewrite $(3.46)$ as

$$
\begin{gathered}
K_{l l^{\prime}}^{\Lambda}\left(\tau, \tau^{\prime} \mid \eta\right)=Z(\Lambda \mid \eta)^{-2} \int_{\mathcal{H}_{\hat{\beta}, \Lambda}} \int_{\mathcal{H}_{\hat{\beta}, \Lambda}} \frac{\phi_{l}(\tau)-\phi_{l}^{\prime}(\tau)}{\sqrt{2}} \cdot \frac{\phi_{l^{\prime}}\left(\tau^{\prime}\right)-\phi_{l^{\prime}}^{\prime}\left(\tau^{\prime}\right)}{\sqrt{2}} \times \\
\times e^{-\hat{V}\left(\phi_{\Lambda}\right)-\hat{V}\left(\phi_{\Lambda}^{\prime}\right)} e^{\frac{J}{2} \sum_{\left\langle l, l^{\prime}\right\rangle}^{\partial \Lambda} \int_{0}^{\hat{\beta}} d \tau\left(\phi_{l}(\tau)-\phi_{l}^{\prime}(\tau)\right) \eta_{l^{\prime}}(\tau)} d \tilde{\mu}_{0}^{\Lambda}\left(\phi, \phi^{\prime} \mid 0\right) .
\end{gathered}
$$

After change of variables:

$$
x_{l}(\tau)=\frac{\phi_{l}(\tau)-\phi_{l}^{\prime}(\tau)}{\sqrt{2}}, \quad y_{l}(\tau)=\frac{\phi_{l}(\tau)+\phi_{l}^{\prime}(\tau)}{\sqrt{2}}
$$

by definition of the measures it is clear that

$$
d \tilde{\mu}_{0}^{\Lambda}\left(\phi, \phi^{\prime} \mid 0\right)=d \tilde{\mu}_{0}^{\Lambda}(x, y \mid 0) .
$$

Then, using definition (3.44) we get:

$$
\begin{aligned}
K_{l l^{\prime}}^{\Lambda}\left(\tau, \tau^{\prime} \mid \eta\right) & =Z(\Lambda \mid \eta)^{-2} \int_{\mathcal{H}_{\hat{\beta}, \Lambda}} \int_{\mathcal{H}_{\hat{\beta}, \Lambda}} x_{l}(\tau) x_{l^{\prime}}\left(\tau^{\prime}\right) e^{-\tilde{V}\left(x_{\Lambda} \mid y_{\Lambda}\right)} \times \\
& \times e^{\frac{J}{2} \sum_{\left\langle l, l^{\prime}\right\rangle}^{\partial \Lambda} \int_{0}^{\hat{\beta}} d \tau y_{l}(\tau) \eta_{l^{\prime}}(\tau)} d \tilde{\mu}_{0}^{\Lambda}\left(x, x^{\prime} \mid 0\right) .
\end{aligned}
$$

Define the measure 


$$
\tilde{\mu}^{\Lambda}\left(d x_{\Lambda} \mid y_{\Lambda}\right)=\tilde{Z}\left(y_{\Lambda}\right)^{-1} e^{-\tilde{V}\left(x_{\Lambda} \mid y_{\Lambda}\right)} d \mu_{0}^{\Lambda}\left(x_{\Lambda} \mid 0\right)
$$

Then

$$
\begin{aligned}
K_{l l^{\prime}}^{\Lambda}\left(\tau, \tau^{\prime} \mid \eta\right)=Z(\Lambda \mid \eta)^{-2} & \int_{\mathcal{H}_{\hat{\beta}, \Lambda}} d \mu_{0}^{\Lambda}\left(y_{\Lambda} \mid 0\right) \tilde{Z}\left(y_{\Lambda}\right) e^{\frac{J}{2} \sum_{\left\langle l, l^{\prime}\right\rangle}^{\partial \Lambda} \int_{0}^{\hat{\beta}} d \tau y_{l}(\tau) \eta_{l^{\prime}}(\tau)} \times \\
& \times\left\langle x_{l}(\tau) x_{l^{\prime}}\left(\tau^{\prime}\right)\right\rangle_{\tilde{\mu}^{\Lambda}\left(\cdot \mid y_{\Lambda}\right)} .
\end{aligned}
$$

Now the proof follows from Lemma 3.4, or more explicitly from (3.33) with $\mathcal{A}_{B_{1}}=x_{l}$ and $\mathcal{A}_{B_{2}}=x_{l^{\prime}}$.

\section{External field analyticity of the Gibbs state}

In the previous sections we have proved uniqueness of the equilibrium state for our model (in the DLR-sense) in the set of tempered EGM, see (3.34). In this section we consider the model (2.1), (2.2) in the presence of external field $h$ :

$$
H^{\Lambda}(h)=H_{0}^{\Lambda}+V^{\Lambda}+\sum_{j \in \Lambda} h \cdot q_{j}, \quad h \in \mathbb{R}^{d} .
$$

Here $H_{0}^{\Lambda}$ and $V^{\Lambda}$ are defined by (2.1)-(2.3). After rescaling (2.6) one gets:

$$
\hat{H}^{\Lambda}(h)=\hat{H}_{0}^{\Lambda}+\hat{V}^{\Lambda}+\sum_{j \in \Lambda} \hat{h} \cdot x_{j}, \quad \hat{h}=m^{-1 / 4} h .
$$

Then the following statement is true:

Theorem 5.1 For the system of quantum particles with Hamiltonian (5.1) there is $m_{*}$, such that for any $0<m<m_{*}, T \geq 0$ and $\left|h^{(\alpha)}\right|<h_{0}\left(m_{*}\right), \alpha=$ $1, \ldots, d$, the limit

$$
\lim _{\Lambda \nearrow \mathbb{Z}^{\nu}} \rho_{\Lambda}^{\beta, h}(A)=\rho^{\beta, h}(A), \quad A \in \mathfrak{A}_{0},
$$

exists. The limit $\rho^{\beta, h}(A)$ gives a unique state on the algebra $\mathfrak{A}_{0}$, which can be continuously extended to the algebra $\mathfrak{A}$. Moreover, the functions $\rho^{\beta, h}(A), A \in$ $\mathfrak{A}$ are analytic in the domain $\left\{h^{(\alpha)} \in \mathbb{C}^{d}|| h^{(\alpha)} \mid<h_{0}\left(m_{*}\right)\right\}$. 
Proof. The proof is close to that of Theorem 2.1. The only remark one has to do is that in expansions (3.20)-(3.27) the factor $\exp \left[-\hat{V}\left(\phi_{X_{n}}\right)\right] \exp \left[\hat{h} \int_{X_{n}} \phi(t) d t\right]$ appears instead $\exp \left[-\hat{V}\left(\phi_{X_{n}}\right)\right]$ and the operator $\Delta(\eta, \bar{Y})$ in (3.22) acts on the product $\mathcal{A}_{B}\left(\phi_{B}\right) \exp \left[-\hat{V}\left(\phi_{X_{n}}\right)\right] \exp \left[h_{m} \int_{X_{n}} \phi(t) d t\right]$. When the derivatives from $\Delta(\eta, \bar{Y})$ act on the factor $\exp \left[\hat{h} \int_{X_{n}} \phi(t) d t\right]$, the convergence is guaranteed by the small factors $h=m^{1 / 4} \hat{h}$ from $\left\{h \in \mathbb{C}^{d}|| h^{(\alpha)} \mid<h_{0}\left(m_{*}\right), \alpha=\right.$ $1, \ldots, d\}$. In principle this implies a new value of the light-mass threshold $m_{*}^{h}$ :

$$
m_{*}^{h}=\min \left\{m_{*}, m_{*}\left(h C_{G} e^{c+1}\right)^{-4}\right\},
$$

where $m_{*}$ is defined in (4.22), $C_{G}$ is from the estimate of the product of covariances in $\Delta(\eta, \bar{Y}), e^{c}$ is from the estimate of $F_{n}^{T}(\bar{Y})$, and additional $e$ is from the inequality (cf. [21]):

$$
\sum_{\eta} \int_{0}^{1}(d s)^{n-1} f\left(\eta ;(s)_{n-2}\right) \leq e^{n}, \quad n=|\eta| .
$$

But by virtue of (5.3) there is $h_{0}\left(m_{*}\right)$ such that $m_{*}^{h}=m_{*}$ for $\left|h^{(\alpha)}\right|<h_{0}\left(m_{*}\right)$, which finishes the proof.

As a consequence of the above theorem we obtain the following result (cf. [43]):

Theorem 5.2 Let $\Lambda_{n}$ be the sequence $\Lambda_{1} \subset \Lambda_{2} \subset \ldots \subset \Lambda_{n} \ldots, \cup_{n=1}^{\infty} \Lambda_{n}=\mathbb{Z}^{\nu}$, and let $h=e\|h\|$, where $e$ is a unit vector in $\mathbb{R}^{d}$. By

$$
\sigma(\beta):=\lim _{h \rightarrow 0} \lim _{n \rightarrow \infty} \rho_{\Lambda}^{\beta, h}\left(\frac{1}{\left|\Lambda_{n}\right|} \sum_{j \in \Lambda_{n}} q_{j} \cdot e\right)
$$

we define the order parameter for the model (5.1). Then there exists mass $m_{*}>0$ such that for any $0<m<m_{*}$ and for all temperatures, including $\beta=\infty$, the order parameter is trivial for any tempered boundary condition defined in (3.34), i.e.:

$$
\sigma(\beta)=0 .
$$


Proof. The proof follows directly from the Theorem 5.1 and cluster expansion for the state

$$
\hat{\rho}_{\Lambda}^{\hat{\beta}, \hat{h}}\left(\frac{1}{\left|\Lambda_{n}\right|} \sum_{j \in \Lambda_{n}} x_{j} \cdot e\right) .
$$

Indeed, if derivatives from $\Delta(\eta, \bar{Y})$ act on the factor

$$
\exp \left\{\int_{X_{n}} \hat{h} \cdot \phi(t) d t\right\}
$$

then after thermodynamic limit the corresponding terms are proportional to $h=m^{-1 / 4} \hat{h}$. If derivatives act on the factor $\phi_{l} \exp \left[-\hat{V}\left(\phi_{X_{n}}\right)\right]$, then in the limit we obtain a Gaussian integration with zero mean and with odd numbers of fields. So, taking into account that $\rho^{\beta, h}$ is analytic in $h$ (Theorem 5.1), in the limit $h=0$ we get zero value (5.5) for the order parameter.

\section{Convergence of cluster expansions and Gibbs state uniqueness for high temperatures}

The problem of the existence and uniqueness of the quantum state (EGM) at high temperatures for the model (2.1)-(2.3) can be solved in the framework of a general approach, developed e.g. in $[13,14]$. But there exist an independent question of construction of high temperature expansions for quantum state (2.5) and of the proof of its convergence in thermodynamic limit. This question is related to a specificity of the one-site potential (2.3) behaviour at infinity. For the quantum systems with unbounded spins the corresponding expansions were considered in $[35,36,45,46,53]$. All these results were obtained for the models with the one-site polynomial anharmonic potentials of the form (1.3). Then the convergence is insured by the small parameter $\beta^{(s-1) / 2 s}$. Since in (2.1)-(2.3) we have $s=1$, there is no such parameter in our case. We recall that $2 s$ is degree of one-site anharmonic polynomial interaction in (1.3).

In the present section we show that approach we developed in Sections 3-5 (the choice of reference measure and the type of cluster expansion) can be adapted to obtain convergent cluster expansions in powers of $\beta>0$, and 
to prove analyticity of the state in domain $\left|\beta-\beta_{*} / 2\right|<\beta_{*} / 2$ of the complex values of $\beta$ around the point $\beta_{*} / 2>0$.

The construction of cluster expansions is absolutely the same as above, but instead of partition of the interval $[0, \beta]=S_{\beta}$ into unit intervals we consider it as one unit with variable $\tau$ running in $S_{\beta}$. In another words, instead of "rods" $\Delta_{j, \hat{\tau}}$ (see (3.5)) we consider for every site $j \in \Lambda$ we consider "rods" $\Delta_{j, \beta}:=\left(j, S_{\beta}\right)$. Then the measure $d \mu_{0 ;(s)_{n}}^{\Lambda}$ is constructed in the same way as in Section 3 with help of the covariance $G_{0 ; j, k}^{\Lambda}\left(\tau, \tau^{\prime} ;(s)_{n}\right)$, see $(3.12)$, where $X_{n}=\emptyset$ and $T=\Lambda$. Then following the line of reasoning of Section 3 we obtain:

$$
\left\langle\mathcal{A}_{B}(\cdot)\right\rangle_{\mu^{\Lambda}}=\sum_{n=1}^{n_{\Lambda}} \sum_{\bar{Y} \subset \Lambda} \mathcal{K}_{\bar{Y}}^{\Lambda}\left(\mathcal{A}_{B}\right) F_{n}^{\Lambda}(\bar{Y})
$$

with the same definitions as in (3.24)-(3.30), and with only difference that

$$
\Delta_{p, p^{\prime}}^{(\alpha)}=\int_{0}^{\beta} d \tau \int_{0}^{\beta} d \tau^{\prime} G_{0 ; Y_{p}, Y_{p^{\prime}}}^{\Lambda}\left(\tau, \tau^{\prime}\right) \frac{\delta^{2}}{\delta \phi_{Y_{p}}^{(\alpha)}(\tau) \delta \phi_{Y_{p^{\prime}}}^{(\alpha)}\left(\tau^{\prime}\right)},
$$

and $Y_{p}, Y_{p^{\prime}}$ are the sites of the lattice $\mathbb{Z}^{\nu} \cap \Lambda$. The main result then is the following

Theorem 6.1 For the quantum system with Hamiltonian (2.1)-(2.3) there exists a sufficiently small (independent of mass $m$ ) value of inverse temperature $\beta_{*}$ such that for all $0<\beta<\beta_{*}$ the weak limit of the measures

$$
\lim _{\Lambda \nearrow \mathbb{Z}^{\nu}} \mu^{\Lambda}=\mu
$$

exists and $\mu$ is a unique translation-invariant Gibbs measure on $\left(\Omega_{\beta, \mathbb{Z}^{\nu}}, \Sigma_{\beta, \mathbb{Z}^{\nu}}\right)$ in the set of tempered measures. Here $\mu^{\Lambda}$ as in Theorem 2.2.

Proof. It is very close to the proof of Theorem 2.2. The only difference is that in the estimate (4.20) one should take

$$
\left(1+\frac{\delta_{m}}{2 \sqrt{a}} \cdot \frac{e^{\hat{\beta} \sqrt{a}}+1}{e^{\hat{\beta} \sqrt{a}}-1}\right)^{-d / 2} \leq\left(\frac{2 \hat{\beta} \sqrt{a}}{2 \hat{\beta} \sqrt{a}+\delta_{m}}\right)^{d / 2} \leq\left(\frac{2 \beta \sqrt{a}}{\delta}\right)^{d / 2} .
$$


This gives for the small parameter of cluster expansion the value

$$
\varepsilon=64 b \sqrt{a} C_{G} \beta^{d / 2} .
$$

Therefore,

$$
\beta_{*}=\left(64 b \sqrt{a} C_{G} e^{c}\right)^{-2 / d},
$$

\section{Conclusions}

In the present paper we construct the Gibbs states for quantum crystal with nonpolynomial anharmonic potential (1.5) and with nearest neighbour harmonic interaction in the light-mass regime. These states are constructed on the algebra of quasi-local observables described in Section 2. Our analysis is based on a reformulation of the quantum Gibbs states problem into the study of Euclidean Gibbs Measures (EGM) [2,7,31,33].

The main technical tool we use to get the main result is the cluster expansions for EGM. They allow us (Theorem 2.1) to prove the existence and uniqueness of translation-invariant EGM in the light-mass domain, for all temperatures $T$, including $T=0(\beta=\infty)$.

To prove the existence of a unique translation-invariant measure we start with periodic boundary conditions (Section 2). Then we prove convergence of the corresponding cluster expansions and the cluster property of the EGM in the light-mass domain for all temperatures $T$. This gives the uniqueness in the class of translation-invariant measures for small mass $m<m_{*}$ and all $T \geq 0$. Next we establish that convergence of the EGM cluster expansions and their cluster properties hold also for zero boundary conditions. This again implies uniqueness in this case. Finally, following the arguments of $[6,11]$ we prove the uniqueness of EGM on the set of tempered configurations (Section 3).

Acknowledgments. We would like to thank S. Albeverio, Yu. Kondratiev, Yu. Kozitsky, and M. Röckner for sending us their article [6] prior to publication and for fruitful discussions. One of the author (A.L.R.) would like to thank the Centre de Physique Théoretique, CNRS-Luminy for the warm hospitality during his stay in October-January of 2002-2003. 


\section{References}

1. V.L. Aksenov, N.M. Plakida, and S. Stamenković, Neutron Scattering by Ferroelectrics, World Scientific, Singapore 1990.

2. S. Albeverio and R. Høegh-Krohn, Homogeneous random fields and quantum statistical mechanics, J. Funct. Anal., 19, 242-272 (1975).

3. S. Albeverio, Yu. Kondratiev, and Yu. Kozitsky, Suppression of Critical Fluctuations by Strong Quantum Effects in Quantum Lattice Systems, Commun. Math. Phys., 194, 493-512 (1998).

4. S. Albeverio, Yu. Kondratiev, Yu. Kozitsky, and M. Röckner, Uniqueness for Gibbs mearsures of quantum lattices in small mass regime, Ann. Inst. H. Poincare, 37, 43-69 (2001).

5. S. Albeverio, Yu. Kondratiev, Yu. Kozitsky, and M. Röckner, Gibbs states of quantum crystal: uniqueness by small particle mass. C.R. Acad. Sci. Paris, Ser. I, 335, 693-698 (2002).

6. S. Albeverio, Yu. Kondratiev, Yu. Kozitsky, and M. Röckner, Small mass implies uniqueness of Gibbs states of a quantum crystal, Commun. Math. Phys., 241, 69-90 (2003).

7. S. Albeverio, Yu. Kondratiev, Yu. Kozitsky, and M. Röckner, Euclidean Gibbs states of Quantum Lattice Systems, Rev. Math. Phys., 14, 13351401 (2002).

8. S. Albeverio, Yu.G. Kondratiev, R.A. Minlos, and A.L. Rebenko, Small mass behaviour of quantum Gibbs states for lattice models with unbounded spins, J. Stat. Phys., 92, 1153-1172, 1998.

9. S. Albeverio, Yu.G. Kondratiev, R.A. Minlos, and G. V. Shchepan'uk, Uniqueness Problem for Quantum Lattice Systems with Compact Spins, Lett. Math. Phys., 52, 185-195, 2000.

10. S. Albeverio, Yu. Kondratiev, T. Pasurek, and M. Röckner, Gibbs states on loop lattice: existence and a priori estimates, C. R. Acad. Sci. Paris, 333, Serie I 1005-1009 (2001) 
11. S. Albeverio, Yu. Kondratiev, T. Pasurek, and M. Röckner, A priori estimates and existence for Euclidean Gibbs measures, Preprint, BiBoS, Nr 02-06-089, Bielefeld (2002)

12. S. Albeverio, A.Yu. Kondratiev, and A.L. Rebenko, Peierls argument and long-range order behaviour of quantum lattice systems with unbounded spins, J. Stat. Phys., 92, 1137-1152, 1998.

13. S. Albeverio, Yu.G. Kondratiev, M. Röckner, and T.V. Tsikalenko, Uniqueness of Gibbs States for Quantum Lattice Systems, Probab. Theory Relat. Fields, 108, 193-218 (1997).

14. S. Albeverio, Yu.G. Kondratiev, M. Röckner, and T.V. Tsikalenko, Dobrushin's Uniqueness for Quantum Lattice Systems with Nonlocal Interactions, Commun. Math. Phys., 189, 621-630 (1997).

15. V.S. Barbulyak and Yu.G. Kondratiev, Functional Integrals and Quantum Lattice Systems: III Phase transitions. Reports Nat. Acad. Sci. of Ukraine, No 10, 19-21 (1991).

16. G. A. Battle III, A new combinatoric estimate for cluster expansions, Commun. Math. Phys., 94, 133-139 (1984).

17. G. A. Battle III and P. Federbush, A note on cluster expansions, tree graph identities, extra $1 / N$ ! factors!!!, Lett. Math. Phys., 8, 55-57 (1984).

18. O. Bratteli and D.W. Robinson, Operator algebras and Quantum Statistical Mechanics, Vol. 2 (second edition), Springer-Verlag, Berlin 1996.

19. A. D. Bruce and R. A. Cowley, Structural phase transitions, Taylor and Francis Ltd, London 1981.

20. D. Brydges, A rigorous approach to Debye screening in dilute classical Coulomb systems, Commun. Math. Phys., 58, 313-350 (1978).

21. D. Brydges and P. A. Federbush, A new form of the Mayer expansion in classical statistical mechanics, J. Math. Phys., 19, 2064-2067 (1978).

22. D. Brydges and P. A. Federbush, Debay Screneeng, Commun. Math. Phys. 73, 197-246 (1980). 
23. V. Cachia and V. A. Zagrebnov, Trotter product formula for nonselfadjoint Gibbs semigroups, J. Lond. Math. Soc., 64, 436-444 (2001).

24. Ph. Choquard, The Anharmonic Crystal, W. A. Benjamin, N.Y. 1967.

25. R.L. Dobrushin, Prescribing a System of Random Variables by Conditional Distributions, Theory Prob. Appl., 15, 458-486 (1970).

26. W. Dressler, L. Landau, and J.F. Perez, Estimates of critical length and critical temperatures for classical and quantum lattice systems, $J$. Stat. Phys., 20, 123-162 (1979).

27. W.G. Faris and R.A. Minlos, A Quantum Crystal with Multidimensional Anharmonic Oscillators, it J. Stat. Phys., 94, 365-387 (1999).

28. J. Ginibre, General Formulation of Griffiths' Inequalities, Commun. Math. Phys., 16, 310-328 (1970).

29. J. Glimm, A. Jaffe, and T. Spencer, The particle structure of the weakly coupled $P(\phi)_{2}$ model and other applications of high temperature expansions. Part II. The cluster expansion. In: it Constructive quantum field theory (eds. G. Velo, A. Wightman). Lecture Notes in Physics 25, Springer-Verlag, Berlin, Heidelberg 1973.

30. J. Glimm and A. Jaffe, Quantum physics. A functional integral point of view, Springer-Verlag, Berlin 1987.

31. S.A. Globa and Yu.G. Kondratiev, The construction of Gibbs states of quantum lattice systems, Selecta Math. Sovietica, 9, 297-307 (1990).

32. R. Høegh-Krohn, Relativistic Quantum Statistical Mechanics in TwoDimensional Space-Time, Commun. Math. Phys., 38, 195-224 (1974).

33. A. Klein and L. Landau, Stochastic processes associated with KMS states, J. Funct. Anal., 42, 368-428 (1981).

34. Yu.G. Kondratiev, Phase transitions in quantum models of ferroelectrics, in: Stochastic proceses, physics and geometry, World Scientific, Singapore, N.Jersey, 465-475 (1994). 
35. A. Yu. Kondratiev and A. L. Rebenko, Some remarks about cluster expansions for ubounded continuous spin systems in quantum statistical mechanics, Methods Funct. Anal. and Topology, 2, 83-99 (1996).

36. A. Yu. Kondratiev and A. L. Rebenko, Cluster expansions of BrydgesFederbush type for quantum lattice systems, Methods Funct. Anal. and Topology, 2, 59-68 (1996).

37. Yu. Kozitsky, Quantum Effects in a Lattice Model of Anharmonic Vector Oscillators, Lett. Math. Phys., 51, 71-81 (2000).

38. Yu. Kozitsky, Scalar Domination and Normal Fluctuation in N-Vector Quantum Anharmonic Crystals, Lett. Math. Phys., 53, 289-303 (2000).

39. Yu. Kozitsky, Quantum Effects in a Lattice Model of Vector Anharmonic Oscillators, in: Stochastic Processes, Physics and Geometry: New Interplays, II (Leipzig, 1999), 403-411, CMS Conf. Proc., 29, Amer. Math. Soc., Providence, RI, (2000).

40. H. Kunz and B. Payandeh, Existence of phase transition for a class of ferroelectric models near the displacive limit, Phys. Rev. B, 18, 2276-2280 (1978).

41. J.L. Lebowitz and E. Presutti, Statistical mechanics of systems of unbounded spins, Commun. Math. Phys., 50, 195-218 (1976).

42. V. A. Malyshev and R. A. Minlos, Gibbs random fields. The cluster expansion method, Kluwer, Dordrecht (1991).

43. R. A. Minlos, E. A. Pechersky, and V. A. Zagrebnov, Analyticity of the Gibbs State for a Quantum Anharmonic Crystal: No Order Parameter, Annal. Henri Poincare, 3, 921-938 (2002).

44. R. A. Minlos, A. Verbeure, and V.A. Zagrebnov, A Quantum Crystal Model in the Light-Mass Limit: Gibbs States, Rev. Math. Phys., 12, 981-1032 (2000).

45. Y. M. Park, The Cluster Expansion for Classical and Quantum Lattice Systems, J. Stat. Phys., 27, 553-576 (1982). 
46. Y.M. Park and H.J. Yoo, A Characterization of Gibbs States for Lattice Boson Systems, J. Stat. Phys., 75, 215-239 (1994).

47. L.A. Pastur and V.A. Khorushenko, Phase transition in quantum models of rotators and ferroelectrics, Theor. and Math. Phys., 73, 111-124 (1987) (English translation).

48. N.M. Plakida and N.S. Tonchev, Exactly soluble $d$-dimensional model of a structural phase transition, Theor. \& Math. Phys., 63, 504-510 (1985).

49. A. L. Rebenko, Mathematical foundations of equilibrium classical statistical mechanics of charged particles, Russ. Math. Surveys, 43, 55-97 (1988).

50. G. Roepstorff, Path Integral Approach to Quantum Physics, SpringerVerlag, Berlin 1991.

51. T. Schneider, H. Beck, and E. Stoll, Quantum effects in an $n$-component vector model for structural phase transitions, Phys. Rev., B 13, 11231130 (1976).

52. B. Simon, Functional Integration and Quantum Physics, Academic Press, New York 1979.

53. W.I. Skrypnik, LRO in lattice systems of linear oscillators with strong bilinear pair nearest-neighbour interaction, J. Phys. A: Math. Gen., 32, 7039-7048 (1999).

54. S. Stamenković, N.S. Tonchev, and V.A. Zagrebnov, Exactly soluble model for structural phase transition with a Gaussian type anharmonicity, Physica A, 145, 262-272 (1987).

55. J. E. Tibballs, R. J. Nelmes, and G. J. McIntyre, The crystal structure of tetragonal $\mathrm{KH}_{2} \mathrm{PO}_{4}$ and $\mathrm{KD}_{2} \mathrm{PO}_{4}$ as a function of temperature and pressure, J. Phys. C: Solid State Phys., 15, 37-58 (1982).

56. V.G. Vaks, Introduction to the microscopic theory of ferroelectrics, Nauka, Moscow 1973 (in Russian). 
57. A. Verbeure and V.A. Zagrebnov, Phase transitions and algebra of fluctuation operators in an exactly soluble model of a quantum anharmonic crystal, J. Stat. Phys., 69, 329-359 (1992).

58. A. Verbeure and V.A. Zagrebnov, No-go theorem for quantum structural phase transitions, J. Phys. A: Math. Gen., 28, 5415-5421 (1995). 